\title{
Resveratrol Impedes the Stemness, Epithelial-Mesenchymal Transition, and Metabolic Reprogramming of Cancer Stem Cells in Nasopharyngeal Carcinoma through p53 Activation
}

\author{
Yao-An Shen, ${ }^{1}$ Chien-Hung Lin, ${ }^{2}$ Wei-Hsin Chi, ${ }^{3}$ Chia-Yu Wang, \\ Yi-Tao Hsieh, ${ }^{3}$ Yau-Huei Wei, ${ }^{1,4}$ and Yann-Jang Chen ${ }^{2,3,5}$ \\ ${ }^{1}$ Institute of Biochemistry and Molecular Biology, National Yang-Ming University, Taipei 112, Taiwan \\ ${ }^{2}$ Institute of Clinical Medicine, National Yang-Ming University, Taipei 112, Taiwan \\ ${ }^{3}$ Department of Life Sciences and Institute of Genome Sciences, National Yang-Ming University, Taipei 112, Taiwan \\ ${ }^{4}$ Department of Medicine, Mackay Medical College, New Taipei City 252, Taiwan \\ ${ }^{5}$ Department of Pediatrics, Taipei City Hospital, Renai Branch, Taipei 106, Taiwan
}

Correspondence should be addressed to

Yau-Huei Wei; joeman@ym.edu.tw and Yann-Jang Chen; yjchen@ym.edu.tw

Received 25 January 2013; Revised 15 March 2013; Accepted 16 March 2013

Academic Editor: Hui-Fen Liao

Copyright (c) 2013 Yao-An Shen et al. This is an open access article distributed under the Creative Commons Attribution License, which permits unrestricted use, distribution, and reproduction in any medium, provided the original work is properly cited.

Cancer stem cells (CSCs) are able to self-renew and are refractory to cancer treatment. To investigate the effects of resveratrol on CSCs of nasopharyngeal carcinoma (NPC), we employed a behavior selection strategy to isolate CSCs based on radioresistance, chemoresistance, and tumor sphere formation ability. These NPC CSCs displayed stem cell properties and underwent metabolic shift to predominately rely on glycolysis for energy supply. Intriguingly, we found that resveratrol turned off the metabolic switch, increased the reactive oxygen species (ROS) level, and depolarized mitochondrial membranes. These alterations in metabolism occurred concomitantly with the suppression of CSC properties including resistance to therapy, self-renewal capacity, tumor initiation capacity, and metastatic potential in NPC CSCs. We found that resveratrol impeded CSC properties through the activation of p53 and this effect could be reversed by knockdown of p53. Furthermore, resveratrol suppressed the stemness and EMT through reactivating p53 and inducing miR-145 and miR-200c, which were downregulated in NPC CSCs. In conclusion, we demonstrated that resveratrol employed the p53 pathway in regulating stemness, EMT, and metabolic reprogramming. Further investigation of the molecular mechanism of p53 activation by resveratrol may provide useful information for the development of novel therapies for cancer treatment through targeting to CSCs.

\section{Introduction}

Nasopharyngeal carcinoma (NPC) is a distinctive type of head and neck cancer with high prevalence rates in Southeast China and Taiwan. Unlike other head and neck cancers, most cases of NPC have high tendency to invade surrounding tissues and metastasize to regional lymph nodes at an early stage. Moreover, most mortality of NPC patients is due to local recurrence and distant metastases [1]. Although chemotherapy and radiotherapy can improve survival rate, the prognosis remains poor for patients with relapse or metastatic diseases [2]. Therefore, in order to effectively identify the target of therapy, the underlying mechanisms that lead to NPC recurrence and metastasis must be clarified.

Emerging evidence substantiated the notion that cancer stem cells (CSCs) or tumor-initiating cells are able to selfrenew and regenerate tumor mass. CSCs are refractory to therapy by dint of their quiescent characteristics and expressing ATP-binding cassette transporters [3]. In addition, epithelial-mesenchymal transition (EMT) imparts not only metastasis capacity but also the CSC properties to tumor cells $[4,5]$. Thereby, the discovery of potential drugs targeting at CSCs may solve the clinical curative difficulties such as therapeutic resistance, recurrence, and metastasis. 
Accumulated evidence has shown that phenolic compounds, such as curcumin, epigallocathechin gallate, and resveratrol, may have potential inhibitory effects on CSCs and prevent tumor invasion and metastasis [6-9]. Among natural polyphenols, resveratrol $\left(3,5,4^{\prime}\right.$-trihydroxy-trans-stilbene) is present mostly in red wine that has pharmacological properties including antiaging, anti-inflammation, and antitumor capacity. Recent studies revealed that resveratrol induced NPC cells apoptosis through activating multiple apoptotic pathways [10]. Furthermore, resveratrol also inhibited CSC properties in pancreatic cancer, breast cancer, and glioblastoma [11-13]. Resveratrol could efficiently suppress the invasion and metastasis of tumor cells through reversing the EMT process in lung and breast cancers $[14,15]$. It also reduced the self-renewal capacity and stemness gene signatures of CSCs in head and neck cancers [9].

However, the mechanisms pertaining to resveratrol mediated signaling pathways in CSCs remained unclear. In this study, we aimed to use NPC CSCs as a model to dissect the regulation of resveratrol in stemness, EMT, and metabolic signatures of CSCs and to explore the potential therapeutic targets in NPC CSCs.

\section{Material and Methods}

2.1. Cell Culture. Three human NPC cell lines, TW01, TW06, and HONE-1, were used in this study $[16,17]$. All NPC cell lines were cultured in complete Dulbecco's Modified Eagle Medium (DMEM), supplemented with $1 \%$ sodium pyruvate, $1 \%$ nonessential amino acids, $1 \%$ of penicillin-streptomycin, and $10 \%$ fetal bovine serum (FBS) (Invitrogen, Carlsbad, CA) at $37^{\circ} \mathrm{C}$ with humidified $5 \% \mathrm{CO}_{2}$.

2.2. CSC Behavior Selection. To acquire high ratio of CSCs from NPC cell lines, we developed a behavior selection method according to CSC's characteristics. The parental cells would be selected through irradiation treatment, sphere formation and side population serially. Firstly, irradiation selection was performed as described previously [18]. Radioresistant clones were established after four rounds of $11 \mathrm{~Gy}$ irradiation at $37.9 \mathrm{mGy} / \mathrm{s}$ using Rad Source RS $2000 \mathrm{X}$ ray biological irradiator (Rad Source Technologies, Inc., Suwanee, GA). The radioresistant capacity of these cells was measured by clonogenic assay. Total of $5 \times 10^{3}$ cells were seeded and treated with $10 \mathrm{~Gy}$ irradiation. After 10 days, colonies were fixed with $3: 1$ ratio of methanol and glacial acetic acid, stained with $2 \%$ crystal violet, and counted under a phase-contrast microscope. After the radioresistant phenotype was corroborated, the selected radioresistant cells were then proceed to tumor sphere selection. Tumor sphere selection was performed as described previously [19]. Cells were seeded into $0.4 \%$ soft agar coated petri dish with serumfree DMEM. The soft surface rendered the cells unable to attach and formed tumor spheres after 10 days. Finally, CSCs were isolated with side population selection by using cells from tumor spheres. Side population assay was performed as described previously [20]. Briefly, cell concentration was adjusted to $10^{6}$ cells/mL with DMEM medium supplemented with $2 \%$ FBS and then treated with Hoechst $33342(5 \mu \mathrm{M}$, Sigma-Aldrich) and incubated in a $37^{\circ} \mathrm{C}$ incubator for $90 \mathrm{~min}$. After twice wash with PBS, propidium iodide (PI) $(2 \mu \mathrm{g} / \mathrm{mL}$, Sigma-Aldrich) was added to exclude dead cells. Cells were kept at $4^{\circ} \mathrm{C}$ in the dark before sorting by BD FACSAria flow cytometer (BD Biosciences, San Jose, CA). The side population gating requires control experiments with the ATP-binding cassette (ABC) transporter inhibitor. As a control, cells were incubated with fumitremorgin C (FTC, $10 \mu \mathrm{M}$, Sigma-Aldrich) for $30 \mathrm{~min}$ at $37^{\circ} \mathrm{C}$ prior to and during Hoechst dye staining. FTC would block the ABC transporters from extruding Hoechst dye. The side population cells would exhibit high fluorescence when treated with FTC to block the efflux of Hoechst dye. We then compared the pattern with or without the treatment of FTC to define and isolate side population cells.

2.3. Survival Fraction Analysis. Survival fractions were performed by comparing the number of survival colonies of the control with the test samples under resveratrol treatment $(10,25$, and $50 \mu \mathrm{M}$, Sigma-Aldrich) and irradiation treatment $(2,4,6,8,10$, and 12 Gy), respectively. Surviving fraction was calculated using the number of colonies divided by the number of cells seeded and corrected with the plating efficiency. Plating efficiency was calculated by dividing the number of colonies with the number of cancer cells seeded.

2.4. Soft Agar Assay. The plates were decoated with $1.2 \%$ soft agar as a base. After the agar solidified, $1 \times 10^{4}$ cells with or without $50 \mu \mathrm{M}$ resveratrol treatment were mixed with $0.4 \%$ soft agar and seeded on the base. The colonies grown in soft agar with diameters $>500 \mu \mathrm{m}$ were counted after two weeks.

2.5. Wound Healing Migration Assay. We utilized ibidi culture inserts (ibidi $\mathrm{GmbH}$, Munich, Germany) for wound healing migration assay and performed according to the manufacture's protocol. The insert contains two reservoirs separated by a $500 \mu \mathrm{m}$ thick wall. We placed the culture insert into a 24-well culture plate and added $1 \times 10^{4}$ cells with or without $50 \mu \mathrm{M}$ resveratrol treatment into each reservoir for overnight culture. A $500 \mu \mathrm{m}$ gap was created after removing culture insert. The images of cell migration were captured by a Dino-Lite microscope eye-piece camera (Dino-Lite, Naarden, Netherlands).

2.6. Invasion Assay. For invasion assay, we placed Millicell invasion chamber $(8 \mu \mathrm{m}$ pore size, Millipore, Darmstadt, German) with Matrigel (BD Biosciences) into a 24-well plate. In the upper compartment of the invasion chamber, $1 \times 10^{4}$ cells were seeded and filled with $200 \mu \mathrm{L}$ serum-free DMEM and $50 \mu \mathrm{M}$ resveratrol. In the lower compartment of the invasion chamber, $600 \mu \mathrm{L}$ complete DMEM with 10\% FBS was added. After 24 hours incubation, the invasive cells located on the underside of the filter were fixed with $3: 1$ ratio of methanol and glacial acetic acid, stained with $2 \%$ crystal violet, and counted under a phase-contrast microscope. 
2.7. Real-Time RT-PCR Analysis. Total RNA was extracted with TRIsure reagent (Bioline Reagents Ltd., London, UK). The concentration and purity of total RNA was determined by NanoDrop ND-1000 Spectrophotometer (NanoDrop Technologies, Inc., Wilmington, DE). Real-time PCR was performed using the SensiFAST SYBR Hi-ROX Kit (Bioline) on the ABI StepOnePlus Real-Time PCR machine (Applied Biosystems, Foster City, CA). The primer sequences used in this study were the same as those described previously $[8,21,22]$.

\subsection{Antibodies for Western Blotting and Immunofluorescence} Staining. Antibodies such as anti- $\alpha$-tubulin $(1: 5000$, Abcam, Cambridge, MA), anti-actin (1:5000, Millipore, Darmstadt, German), anti-Oct3/4 (1:1000, Bioworld Technology, Suffolk, UK), anti-E-cadherin (1:5000, BD Biosciences), antivimentin (1:1000, Sigma-Aldrich), anti-p53 (1:1000, Millipore), and anti-phospho-p53 (Ser15) (1:1000, Cell Signaling, Boston, MA) were used for western blotting and immunofluorescence staining. Western blotting and immunofluorescence staining were performed as described previously [8].

2.9. Plasmids, Virus Production, and Infection. Human p53 cDNA was subcloned into pEZ-Lv201-puro lentiviral vector (GeneCopoeia, Rockville, MD, USA). p53 shRNA was cloned in pLKO.1-based lentiviral vector provided by the National RNAi Core Facility, Academia Sinica, Taiwan. We chose the p53 shRNA with the best knockdown efficiency to do further experiment from four p 53 shRNAs targeted against different regions of p53. EGFP was cloned into the pAS2puro-based lentiviral vector provided by the National RNAi Core Facility, Academia Sinica, Taiwan. Utilizing PolyJet DNA In Vitro Transfection Reagent (SignaGen Laboratories, Rockville, MD), 293T cells were transfected with viral vectors. The virus-containing supernatant of transfectant was filtered through a $0.45 \mu \mathrm{m}$ pore size filter 2 days after transfection. Viral infection was achieved by adding virus-containing supernatant supplemented with $8 \mathrm{ng} / \mathrm{mL}$ polybrene. Puromycin selection $(5 \mu \mathrm{g} / \mathrm{mL})$ was subsequently employed 24 hours after infection. After one week of puromycin selection, stable clones were used for further analysis.

2.10. Measurement of Oxygen Consumption Rate. Oxygen consumption rate was measured by the 782 Oxygen Meter (Strathkelvin Instruments, Motherwell, UK). To measure the steady-state oxygen consumption rate of the cells, $1 \times$ $10^{6}$ cells with or without $50 \mu \mathrm{M}$ resveratrol treatment were resuspended in $330 \mu \mathrm{L}$ of assay buffer ( $125 \mathrm{mM}$ sucrose, $65 \mathrm{mM} \mathrm{KCl}, 2 \mathrm{mM} \mathrm{MgCl}_{2}$, and $20 \mathrm{mM} \mathrm{Na}^{+}-\mathrm{K}^{+}$-phosphate buffer, $\mathrm{pH}$ 7.2) and transferred to a closed chamber of the oxygen meter. To measure the function of the mitochondrial respiratory chain, we first permeabilized the mitochondrial outer membrane by adding $0.002 \%$ ethanol-purified digitonin (Sigma-Aldrich) to the chamber (final concentration: $0.0006 \%)$. We then injected $6 \mu \mathrm{L}$ of succinate into the chamber (final concentration: $20 \mathrm{mM}$ ). To assay the efficiency of ATP synthesis, we added $1 \mathrm{mM}$ ADP to the chamber to stimulate the respiration. Oxygen consumption rate was also measured with a Seahorse XF24 extracellular Flux analyzer as described by Qian and Van Houten [23]. Cells were seeded in an XF24 cell culture plate and were allowed to grow for one day. The medium was replaced with unbuffered DMEM and incubated in a $37^{\circ} \mathrm{C}$ incubator for 30 minutes to stabilize the $\mathrm{pH}$ and temperature. The oxygen consumption rate (OCR) represented mitochondrial respiration, and the rate of extracellular acidification (ECAR) indicated the lactate production rate by anaerobic glycolysis.

2.11. Lactate Production Rate. Cells with or without $50 \mu \mathrm{M}$ resveratrol treatment were washed with PBS and replenished with fresh medium in a $37^{\circ} \mathrm{C}$ incubator for 5 hours. An aliquot of $10 \mu \mathrm{L}$ of medium was then transferred to 96 -well dishes and mixed with lactate reagent (Trinity Biotech Plc., Bray, Ireland). The absorbance at $540 \mathrm{~nm}$ was measured by an ELISA reader (PowerWave ${ }^{X} 340$ ). The lactate production rate was calculated from the absorbance and was normalized by the cell number and incubation time.

2.12. Mitochondrial Membrane Potential $(\Delta \psi m)$. Cells with or without resveratrol treatment were trypsinized and incubated with $0.25 \mu \mathrm{g} / \mathrm{mL} \mathrm{JC}-1$ fluorescence dye at $37^{\circ} \mathrm{C}$ in the dark for 15 minutes. The fluorescence intensities of FL1 and FL2 channels were analyzed with Cytomics FC500 flow cytometer (Beckman Coulter, Fullerton, CA).

2.13. Intracellular Levels of Reactive Oxygen Species (ROS). Cells with or without resveratrol treatment were washed by PBS and incubated with a medium containing $40 \mu \mathrm{M} 2^{\prime}, 7^{\prime}$ dichlorofluorescin diacetate $\left(\mathrm{H}_{2} \mathrm{DCFDA}\right.$, Invitrogen $)$ at $37^{\circ} \mathrm{C}$ in the dark for 15 minutes. We then trypsinized the cells and resuspended them in PBS. $\mathrm{H}_{2}$ DCFDA was used for the determination of intracellular $\mathrm{H}_{2} \mathrm{O}_{2}$. The fluorescence intensity of FL1 channel for $\mathrm{H}_{2}$ DCFDA staining was analyzed with Cytomics FC500 flow cytometer (Beckman Coulter).

2.14. Cell Viability Assay. In the drug treatment experiment, cells were exposed to resveratrol $(10,25,50,75$, and $100 \mu \mathrm{M})$, cisplatin $(8 \mu \mathrm{M}$, Sigma-Aldrich), or 5 -fluorouracil (5-FU; $10 \mu \mathrm{M}$, Sigma-Aldrich) for 48 hours. In the irradiation treatment experiment, cells were irradiated (5 Gy or $10 \mathrm{~Gy}$ ) at $37.9 \mathrm{mGy} / \mathrm{s}$ using Rad Source RS 2000 X-ray biological irradiator (Rad Source Technologies, Inc.). After 2 days of culture, cells were washed with PBS, replenished with fresh medium containing $1 \times$ Alamar Blue cell viability assay reagent ( $\mathrm{AbD}$ Serotec, Oxford, $\mathrm{UK}$ ), and incubated at $37^{\circ} \mathrm{C}$ for 2 hours. Fluorescence intensity, with the excitation wavelength at $538 \mathrm{~nm}$ and the emission wavelength at $590 \mathrm{~nm}$, was measured using a Fluoroskan Ascent Microplate Fluorometer (Thermo Fisher Scientific, Inc., Waltham, MA).

2.15. In Vivo Analysis of the Tumor Curability by Resveratrol. To monitor the in vivo growth of CSCs, we first established the GFP-CSCs by transfecting a pAS2-EGFP-puro expression construct to NPC CSCs. A total of 100 GFP-CSCs were then injected into hypoglossal regions of 7-8-week-old NOD/SCID mice. In the group of resveratrol treatment, we fed mice 
with $100 \mathrm{mg} / \mathrm{kg}$ resveratrol every 2 days after injecting the CSCs. The incidence of tumor growth was measured after 2 weeks. The mice were sacrificed, and the distribution of GFPpositive tumors was observed through Youlum Sky-blue II epifluorescent light (Youlum Inc., New Taipei City, Taiwan).

2.16. Statistical Analysis. All data are presented as mean \pm SD. Statistical analysis was performed by using Student's $t$-test and a difference between groups with $P<0.05$ is considered significant.

\section{Results}

3.1. NPC CSCs Exhibit Characteristics of Stemness, EMT, and Metabolic Reprogramming. The most common methods used to isolate CSCs focus on single CSC ability, such as side population or tumor sphere. To improve upon these current methods, we combined irradiation selection, tumor sphere selection, and side population selection to isolate CSCs. The three methods overlapped to form a bull's eye area where purity of CSCs may be the highest (Figure 1(a)). We started with establishing NPC radioresistant clones (Figure 1(b)) by four rounds of high-dose irradiation selection. Several radioresistant NPC clones were established. These radioresistant clones underwent tumor sphere selection and displayed higher tumor sphere formation capacity compared with parental cells (Figure 1(c)). The percentage of side population is greatly higher in tumor sphere cells than that in parental cells (Figure 1(d)). It signified that irradiation selection can increase the feasibility to obtain CSC-like cells as compared with the feasibility of isolating CSC-like cells directly from parental cells merely by tumor sphere or side population selection. Following behavior selection, the CSC clones became resistant to radiotherapy and chemotherapy and capable of forming tumor spheres. Stemness-related transcription factor Oct4 was expressed and translocated from cytoplasm to the nucleus of isolated CSCs (Figure 2(a)). This implied that nuclear Oct 4 might activate its target genes and result in stem cell-like features of CSCs. CSCs also underwent an EMT with downregulation of epithelial marker E-cadherin and concomitant upregulation of mesenchymal marker vimentin (Figures 2(b) and 2(c)). CSCs manifested greater migratory capacity than did parental NPC cells (Figure 2(d)). To know the metabolic characteristics, we then investigated the organelle function of NPC CSCs. The $\mathrm{O}_{2}$ consumption rates driven by ADP and succinate were lower in CSCs compared with those in parental NPC cells (Figure 2(e)), which implied that these CSCs might not rely on mitochondrial respiration to produce energy. Likewise, CSCs excreted higher levels of lactic acid as a by-product than did parental NPC cells (Figure 2(f)). Moreover, the level of prooxidants was relatively lower in CSCs compared with parental NPC cells through analysis of intracellular $\mathrm{H}_{2} \mathrm{O}_{2}$ with $\mathrm{H}_{2}$ DCFH-DA (Figure $2(\mathrm{~g})$ ). These findings revealed that CSCs displayed unique metabolic signatures similar to those of stem cells.

3.2. Resveratrol Suppresses the Stemness, EMT, and Metabolic Reprogramming in CSCs. The versatile efficacy of resveratrol on NPC CSCs was unraveled in this study. We first evaluated the sensitivity of parental cells or CSCs under different concentrations of resveratrol treatment. Cell viability was significantly affected if the concentration of resveratrol was higher than $50 \mu \mathrm{M}$ in parental cells or CSCs (Figure 3(a)). We then mainly used $50 \mu \mathrm{M}$ resveratrol in following assays. Resveratrol could suppress the long-term self-renewal and antianoikis capacity (Figure 3(b)) and in vitro tumorigenicity (Figure 3(c)). Resveratrol also significantly reduced the percentage of side population (Figure 3(d)) and migration and invasion capacity in CSCs (Figures 3(e) and 3(f)). Combining irradiation with resveratrol increased the radiosensitivity of CSCs which suggested that resveratrol could potentially serve as an irradiation sensitizer (Figure $3(\mathrm{~g})$ ). In addition, resveratrol diminished the expression of genes characteristic of stemness, EMT, and metabolism (Figure 3(h)). Downregulation of stemness-related genes such as Oct4, Sox2, Klf4, c-Myc, Nanog, and Lin28 depicted that resveratrol compelled the differentiation of CSCs. Under resveratrol treatment, ABCG2 suppression also increased the drug sensitivity of CSCs. In addition, resveratrol reversed the EMT phenotype of CSCs by inhibiting the expression of EMT regulators and mesenchymal markers, such as Twist, Snail, Zebl, vimentin, and fibronectin; on the contrary, epithelial marker E-cadherin was increased. Moreover, downregulation of pyruvate dehydrogenase kinase (PDK), a negative regulator of pyruvate dehydrogenase (PDH), promoted pyruvate back to mitochondrial biosynthetic pathways in CSCs. As expected, increased $\mathrm{O}_{2}$ consumption and decreased lactate production demonstrated that resveratrol reversed the metabolic shift in NPCCSCs (Figures 4(a) and 4(b)). Resveratrol also significantly increased the ROS level and depolarized mitochondrial membranes in CSCs especially under irradiation treatment (Figures 4(c) and 4(d)).

\subsection{Resveratrol Impedes CSC Properties through p53 Activa-} tion. We found that $\mathrm{p} 53$ expression level was lower in CSCs than that in parental cells (Figure 5(a)). Furthermore, the mesenchymal cell shape of CSCs was reversed to epithelial type concomitant with the activation of p53 (phosphorylated at serine 15) after resveratrol treatment (Figures 5(a) and 5(b)). To confirm the idea that resveratrol could suppress the CSC properties directly through the induction of p53, we modulated the expression of p53 in CSCs by infecting a lentivirus-based full-length p53 clone and shRNAs targeting p53, respectively (Figures 5(c) and 5(d)). We then measured the oxygen consumption rate (OCR) and extracellular acid efflux rate (ECAR) of these CSCs by a Seahorse XF24 Extracellular Flux Analyzer. OCR is an indicator of mitochondrial respiration, and ECAR is predominantly a measure of lactic acid production rate during glycolysis. Similar to the effects of resveratrol, overexpression of $\mathrm{p} 53$ increased the OCR/ECAR ratio in CSCs; knockdown of p53 reduced the OCR/ECAR ratio during resveratrol treatment (Figure 6(a)). These data substantiated the notion that resveratrol altered the energy metabolism mainly by induction of p53. Remarkably, suppression of tumor sphere formation and soft agar colony formation achieved by resveratrol treatment could be rescued 


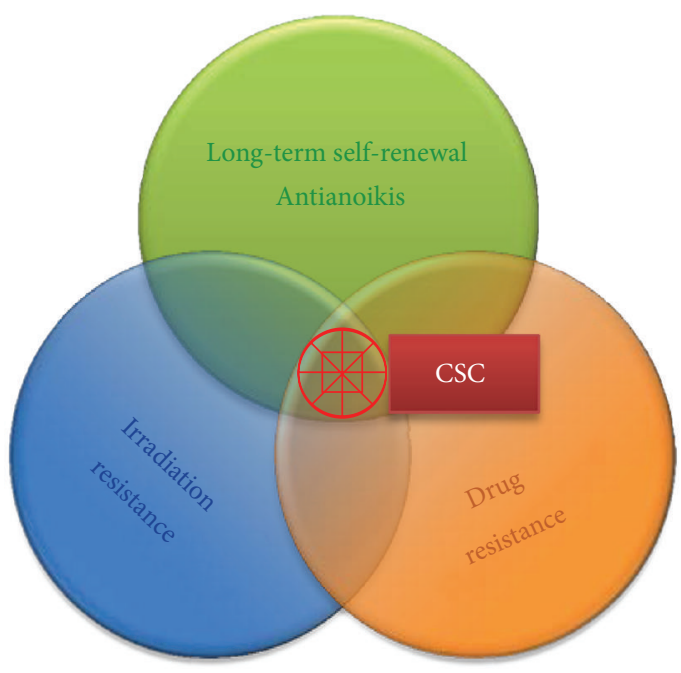

(a)
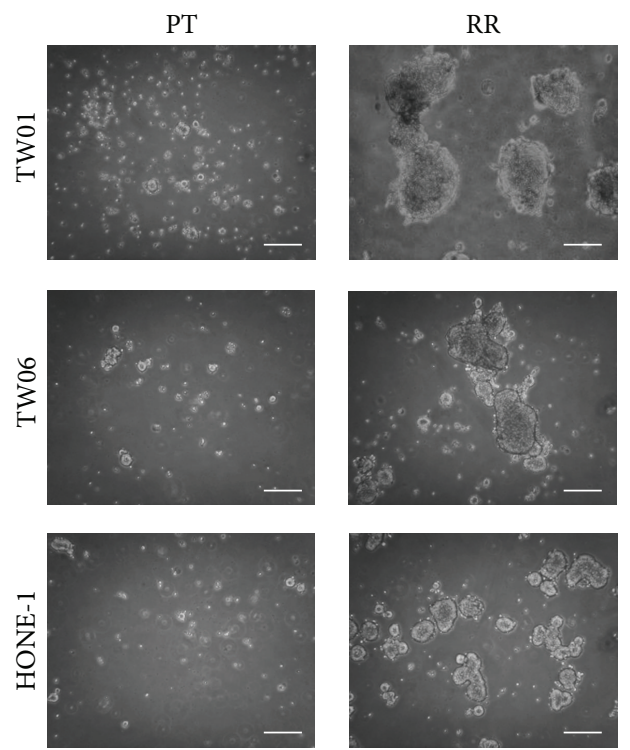

(c)
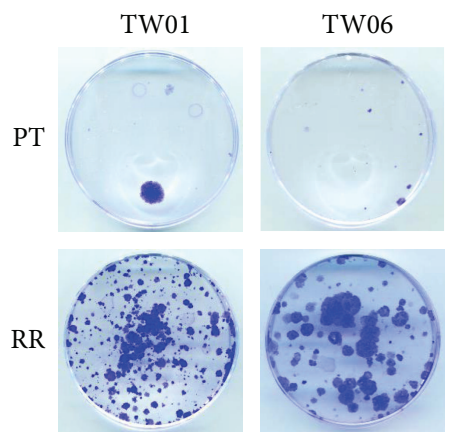

(b)
HONE-1
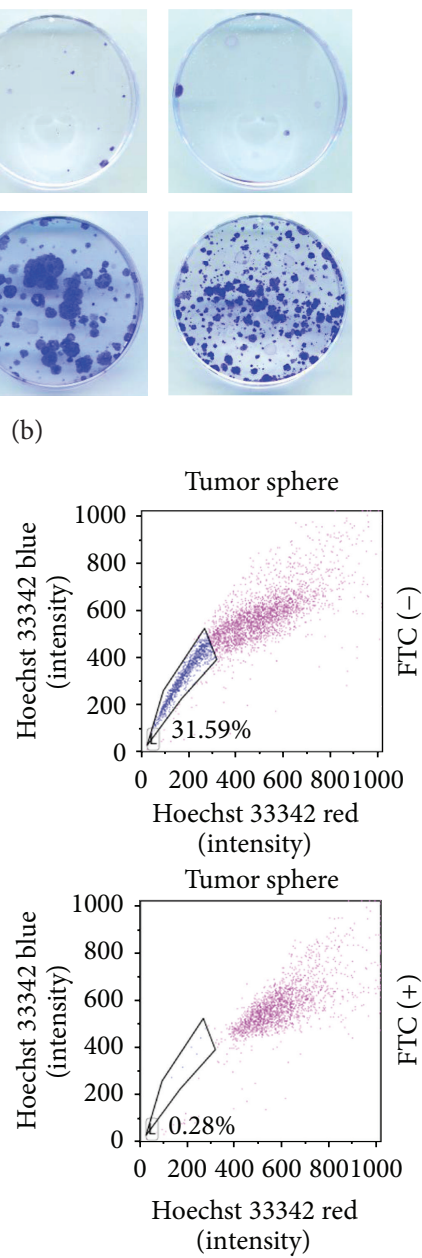

(d)

FIGURE 1: The concept and process validation of NPC CSC isolation achieved by behavior selection. (a) The concept of behavior selection is based on CSC properties, which are resistance to irradiation and drugs, long-term self-renewal, and antianoikis. Through the behavior selection platform, abundant and high-purity CSCs could be obtained. (b) After four rounds of 11 Gy irradiation selection, radioresistant clones (RR) of TW01, TW06, and HONE-1 were established. Further colony formation assays of these radioresistant cells and parental cells (PT) were performed with seeding $5 \times 10^{3}$ cells after 10 Gy irradiation. Representative images are macroscopically visible survival colonies after fixation and crystal violet staining. Radioresistant cells show distinct radioresistant phenotype. (c) After irradiation selection, tumor sphere selection was performed. Compared with parental cells, radioresistant clones could generate more and larger spheres. Scale bars indicate $100 \mu \mathrm{m}$. (d) Side population assay shows that TW01 parental cells (left panel) contained less side population cells compared with tumor sphere cells (right panel). The top panel represents the cells incubated with Hoechst 33342. A subset of side population cells pumped out of the Hoechst dye by $\mathrm{ABC}$ transporters and demonstrated low fluorescence expression. The bottom panel shows that the gating proportion is reduced from $0.15 \%$ to $0.11 \%$ in parental cells and from $31.59 \%$ to $0.28 \%$ in tumor sphere cells after fumitremorgin C (FTC) treatment. FTC can block the $\mathrm{ABC}$ transporters and result in full side population efflux inhibition.

by knocking down p53 (Figures 6(b) and 6(c)). Moreover, the sensitivity to irradiation and chemotherapeutic agents induced by resveratrol could be blocked by knocking down p53 (Figures 6(d) and 6(e)). Invasive capacity was also found to be derepressed after p53 knockdown in resveratroltreated CSCs (Figure 6(f)). As anticipated, resveratrol and p53 overexpression reduced the expression of stemness and EMT, while the silencing of p53 restored the expression of 

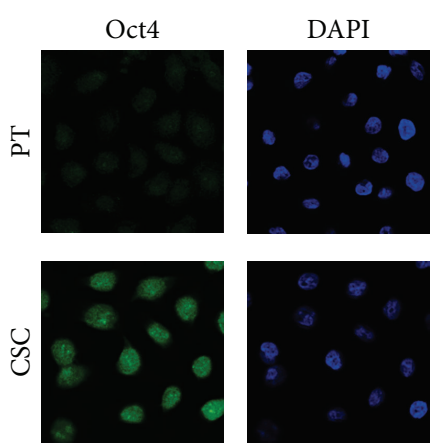

(a)
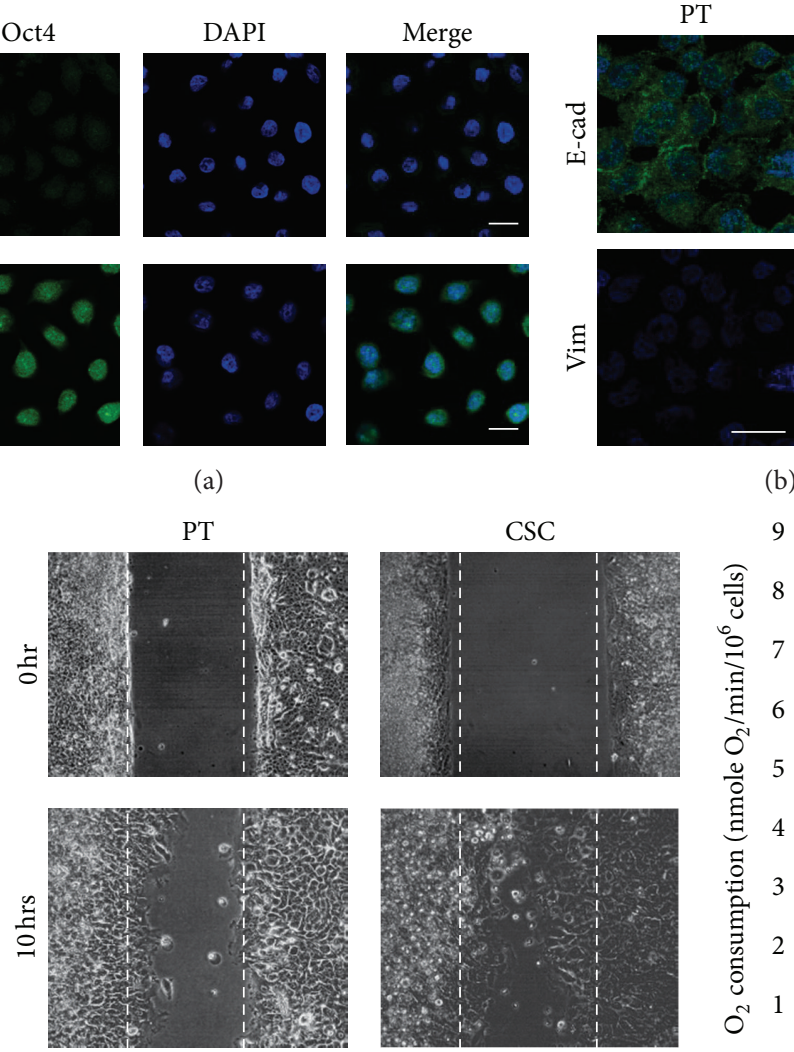

(b)
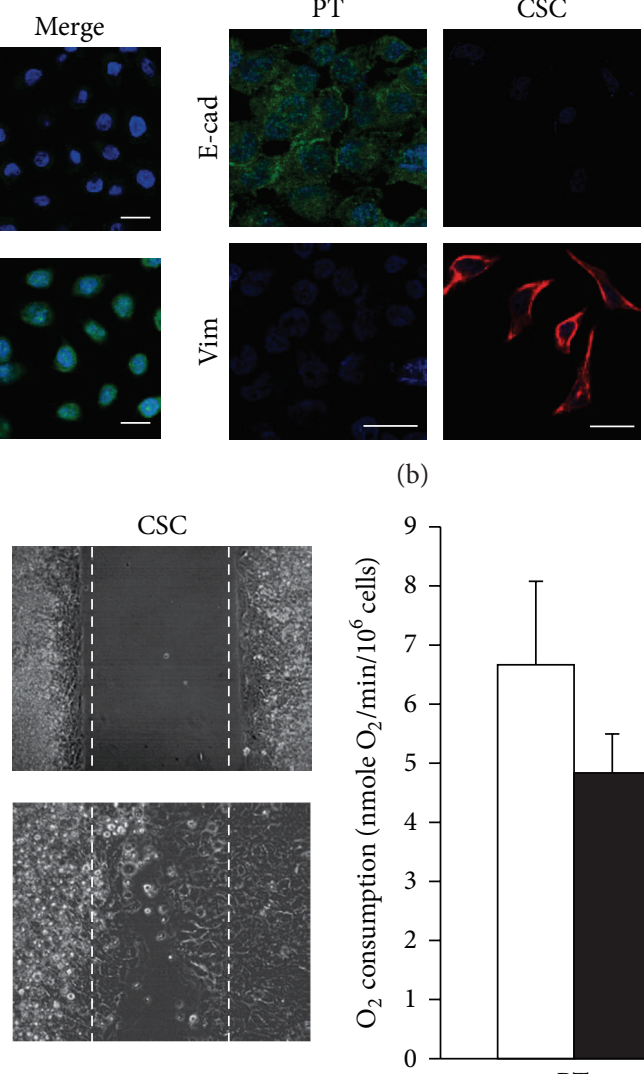

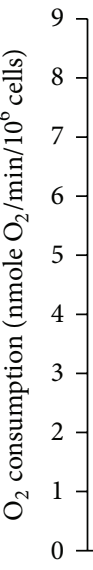

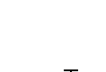

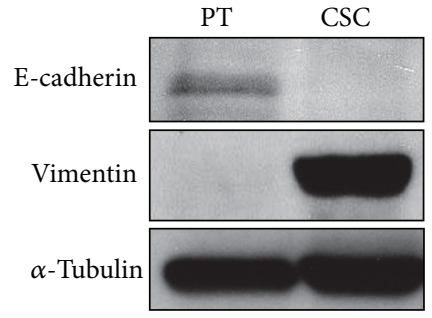

(c)

$\square$ ADP-supported respiration

Succinate-supported respiration

(d)

(e)

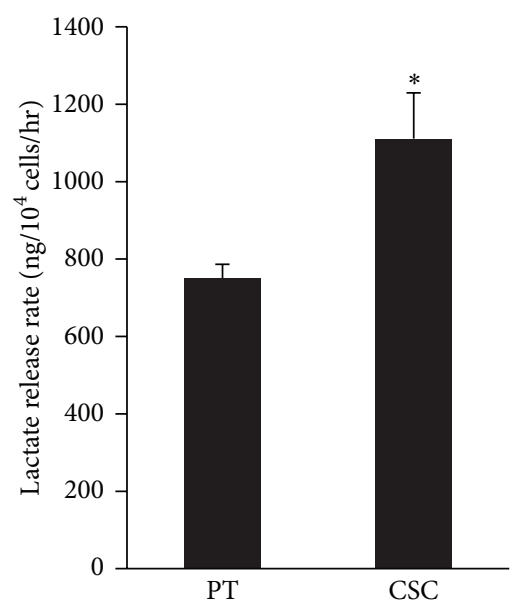

(f)

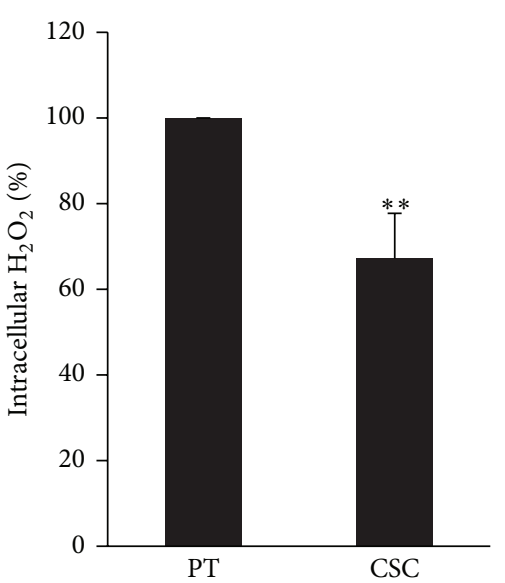

(g)

FIGURE 2: NPC CSCs manifested characteristics of stemness, EMT, and metabolic reprogramming. (a) Images of immunofluorescence staining for Oct4 expression show overexpression and nuclear translocation of Oct4 in TW01 CSCs compared with the negative control of TW01 parental cells. (b) Immunofluorescence staining indicates the transition of epithelial marker (E-cad: E-cadherin) and mesenchymal marker (Vim: Vimentin) in TW01 parental cells and CSCs. Scale bars in (a) and (b) indicate $25 \mu \mathrm{m}$. (c) Western blots confirm the EMT of TW01 CSCs at the protein level. (d) Wound healing assay showed higher migratory behavior of TW01 CSCs compared with TW01 parental cells. Full wound closure was observed in TW01 CSCs after 10 hours. (e) The $\mathrm{O}_{2}$ consumption rates supported by ADP and succinate were lower in TW01 CSCs compared with TW01 parental cells. (f) TW01 CSCs had higher levels of lactate production than did TW01 parental cells. (g) Intracellular $\mathrm{H}_{2} \mathrm{O}_{2}$ level was relatively lower in TW01 CSCs compared with that in TW01 parental cells measured by $\mathrm{H}_{2}$ DCFDA staining. $\left({ }^{*} \mathrm{P}<0.05 ;{ }^{* *} \mathrm{P}<0.01\right)$. 


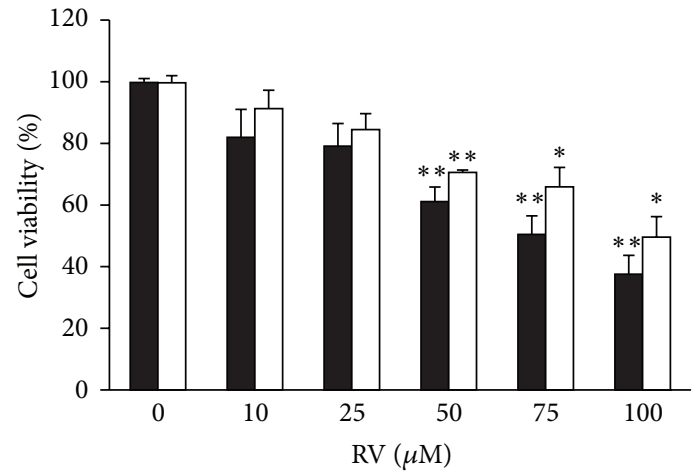

PT

$\square \mathrm{CSC}$

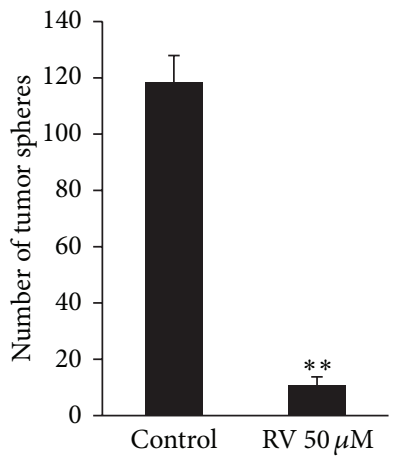

(b)

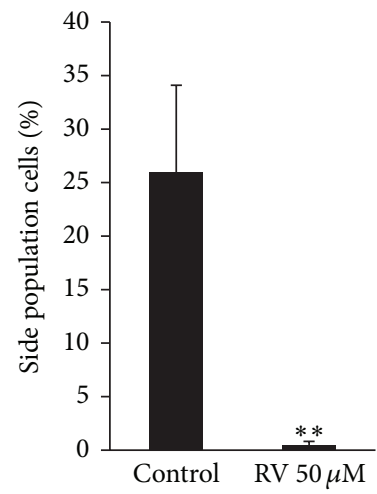

(d)
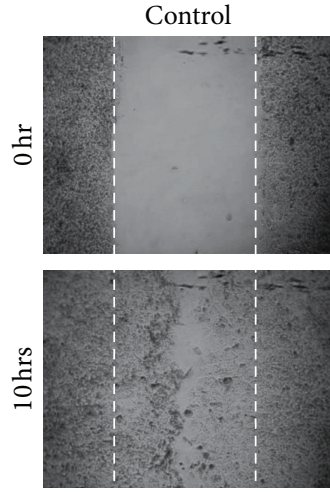

(e)

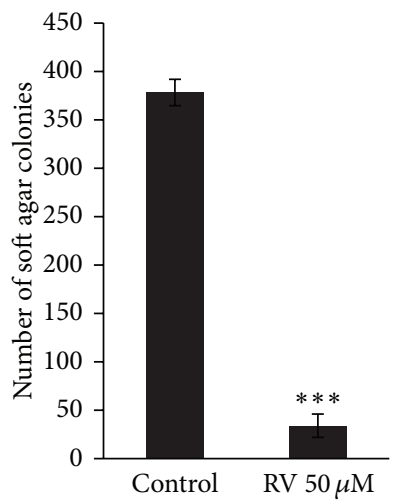

(c)

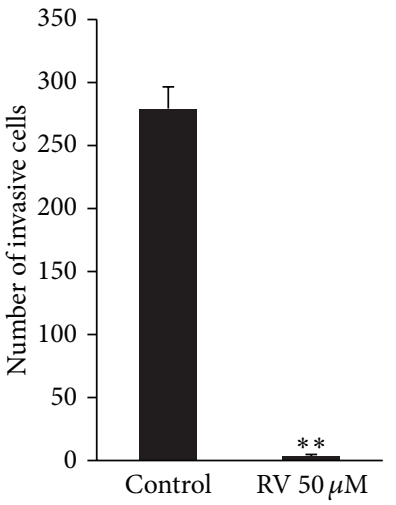

(f)

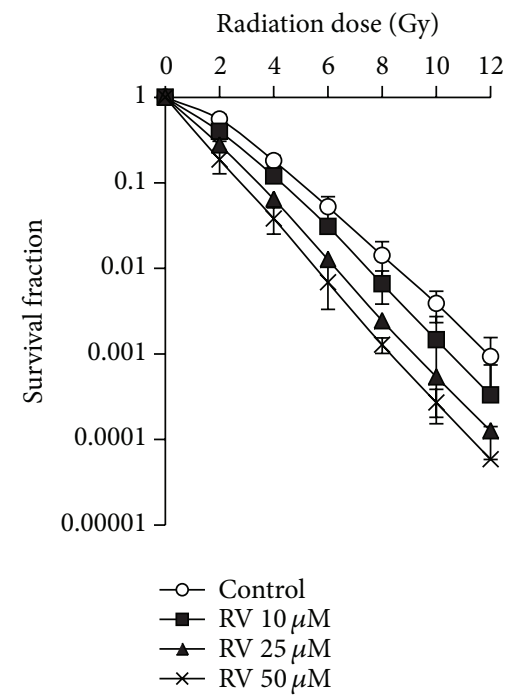

(g)

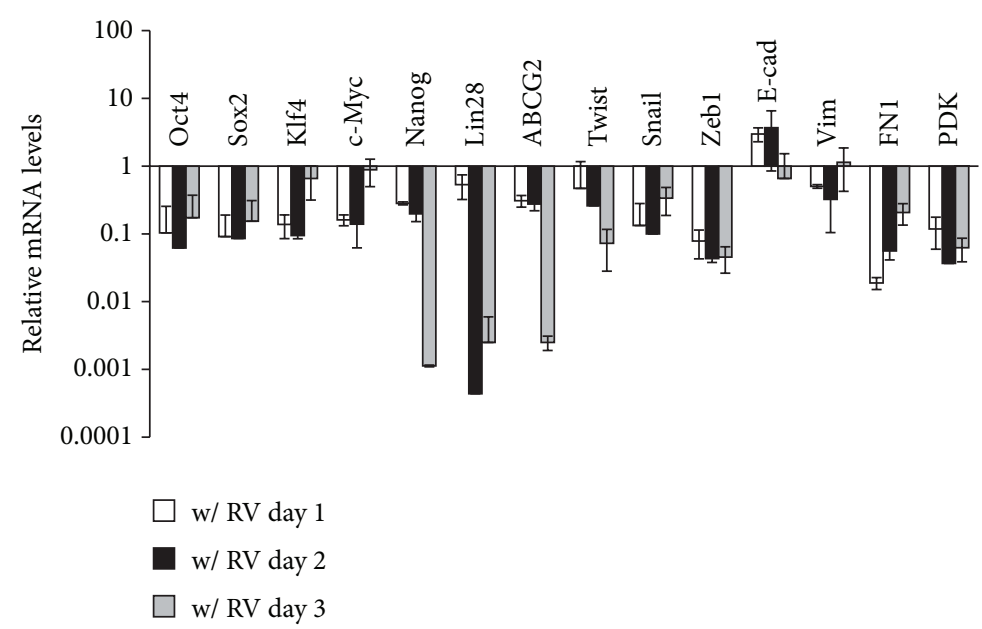

(h)

FIGURE 3: Resveratrol suppressed CSC properties. (a) Evaluation of cytotoxic effects of different concentration of resveratrol (RV) in TW01 parental cells and CSCs. Significant effects are shown with concentration more than $50 \mu \mathrm{M}$. Capacities for (b) tumor sphere formation, (c) soft agar colony formation, and (d) percentage of the side population were suppressed in TW01 CSCs after $50 \mu \mathrm{M}$ resveratrol treatment. (e) Migration capacity analyzed by wound healing assay and (f) invasive capacity measured by transwell invasion assay were suppressed by $50 \mu \mathrm{M}$ resveratrol in TW01 CSCs. (g) Resveratrol could increase the radiosensitivity of TW01 CSCs in a concentration-dependent manner measured by survival fraction assay. (h) Resveratrol $(50 \mu \mathrm{M})$ altered the expression of indicated genes in TW01 CSCs. E-cad: E-cadherin; N-cad: Ncadherin; Vim: vimentin; FN1: fibronectin 1; PDK: pyruvate dehydrogenase kinase. Data were normalized with the mRNA expression level of $18 S$ rRNA and compared with those of TW01 CSCs without resveratrol treatment on a log scale. $\left({ }^{*} P<0.05\right.$; ${ }^{* *} P<0.01$; $\left.{ }^{* * *} P<0.001\right)$. 

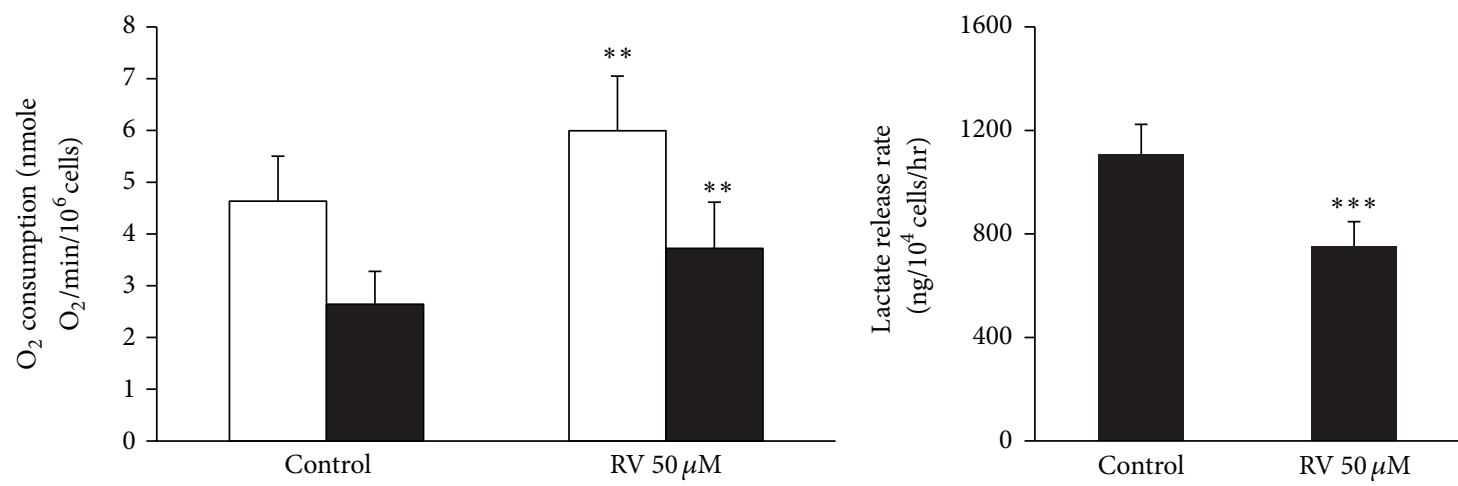

ADP-supported respiration

Succinate-supported respiration

(a)

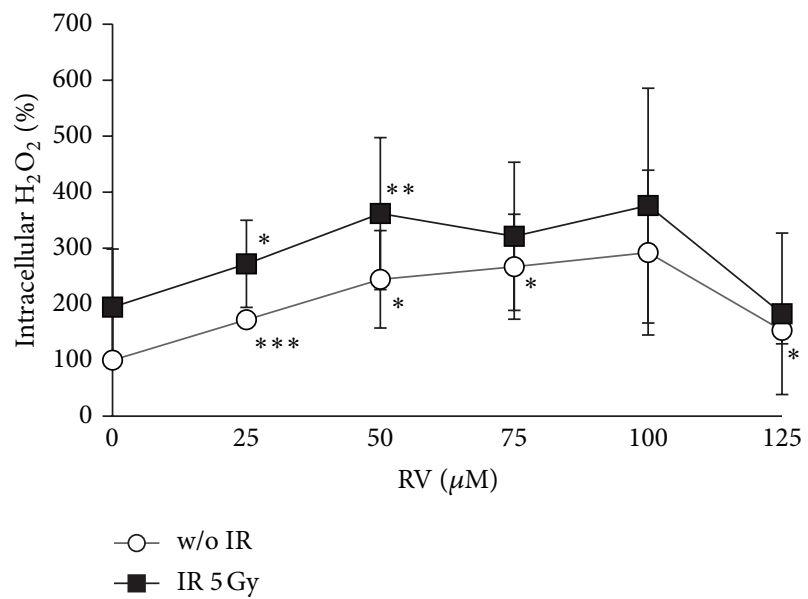

(c) (b)

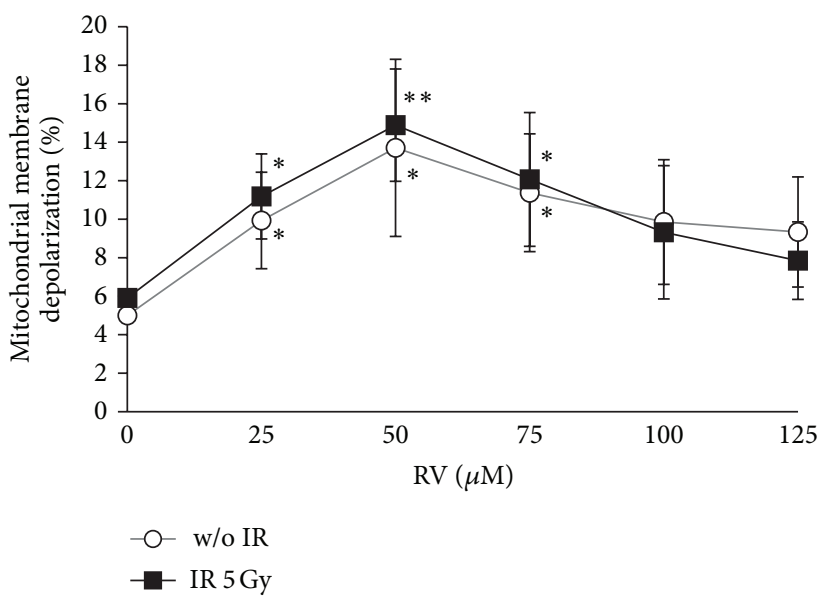

(d)

FIGURE 4: Resveratrol could reverse the metabolic reprogramming of CSCs. (a) Resveratrol $(50 \mu \mathrm{M})$ increased $\mathrm{O}_{2}$ consumption and (b) repressed the lactate production of TW01 CSCs. (c) Intracellular $\mathrm{H}_{2} \mathrm{O}_{2}$ was upregulated in TW01 CSCs exposed to serial doses of resveratrol and 5 Gy irradiation (IR) measured by $\mathrm{H}_{2}$ DCFDA staining. (d) Serial doses of resveratrol depolarized the mitochondrial membrane in TW01 CSCs with or without $5 \mathrm{~Gy}$ irradiation. The percentage of mitochondrial membrane potential depolarization was measured by the JC-1 green fluorescence shift. In (c) and (d) charts, TW01 CSCs with resveratrol treatment were compared with those of TW01 CSCs without resveratrol treatment. TW01 CSCs with both resveratrol and irradiation treatment were compared with those of TW01 CSCs with irradiation but without resveratrol treatment. $\left({ }^{*} \mathrm{P}<0.05 ;{ }^{* *} \mathrm{P}<0.01 ;{ }^{* * *} \mathrm{P}<0.001\right)$.

stemness and EMT in resveratrol-treated CSCs (Figures 6(g) and $6(\mathrm{~h}))$. In addition, we noticed that miR-145 and miR200c were downregulated in CSCs but could be upregulated by resveratrol treatment and p53 overexpression (Figure 6(i)). The upregulation of miR-145 and miR-200c could be attenuated by knockdown of p53 in resveratrol-treated CSCs (Figure 6(i)).

3.4. Resveratrol Annihilates CSCs in NOD/SCID Mice. Lastly, we examined the curative effect of resveratrol in vivo. We injected GFP-CSCs into the hypoglossal region of NOD/SCID mice. The mouse without resveratrol feeding was asthenic in appearance, lost their body weight gradually, and tumor lumps disseminated around the bottom of buccal mucosa (Figures 7(a) and 7(b)). By in vivo GFP tracking, we observed that tumor grew throughout the tongue and lower jaw of the mice without resveratrol treatment (Figure $7(\mathrm{c})$ ). The CSCs were finally eradicated, and the mice stayed healthy when the mice fed with a diet supplemented with resveratrol (Figure $7(\mathrm{~d})$ ).

\section{Discussion}

Metabolism has been the focus of cancer research as early as the 1920s, when Otto Warburg observed that malignant tissues produce energy predominately by glycolysis rather than oxidative phosphorylation (OXPHOS) even when oxygen is plentiful [24]. This metabolic shift from aerobic metabolism to glycolysis also occurs in embryonic stem cells (ESCs) and induced pluripotent stem cells (iPSCs) [25]. Similar 


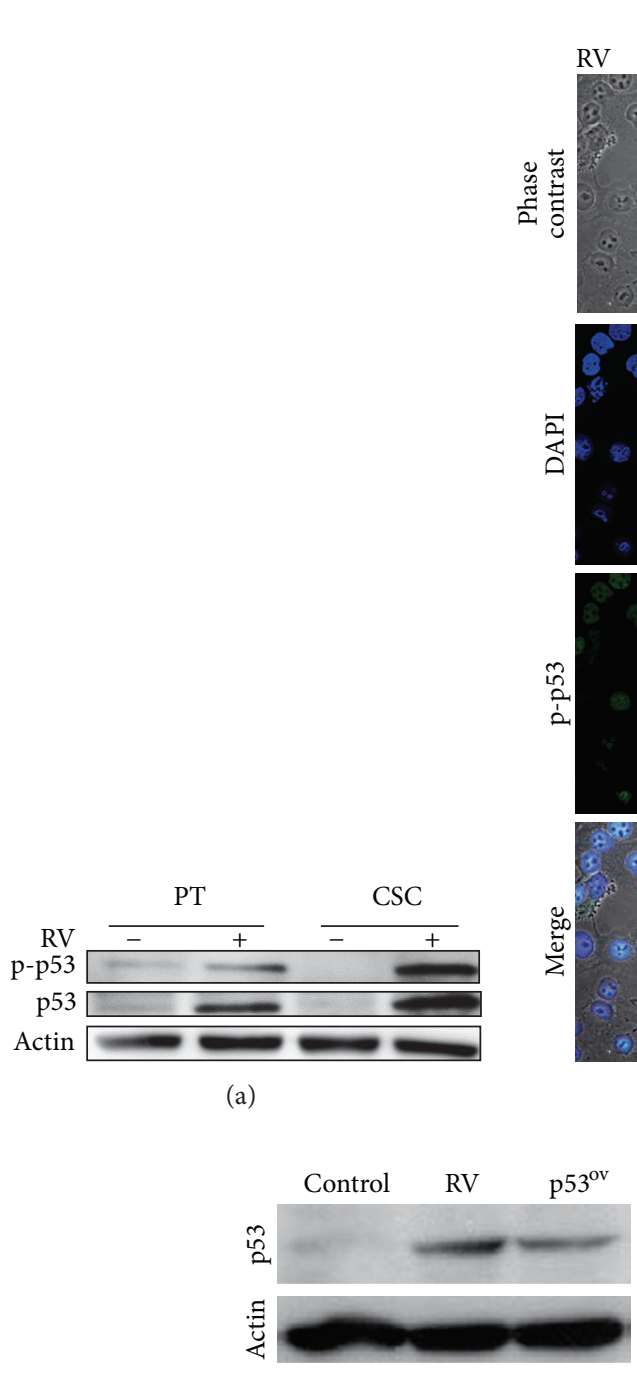

(c)
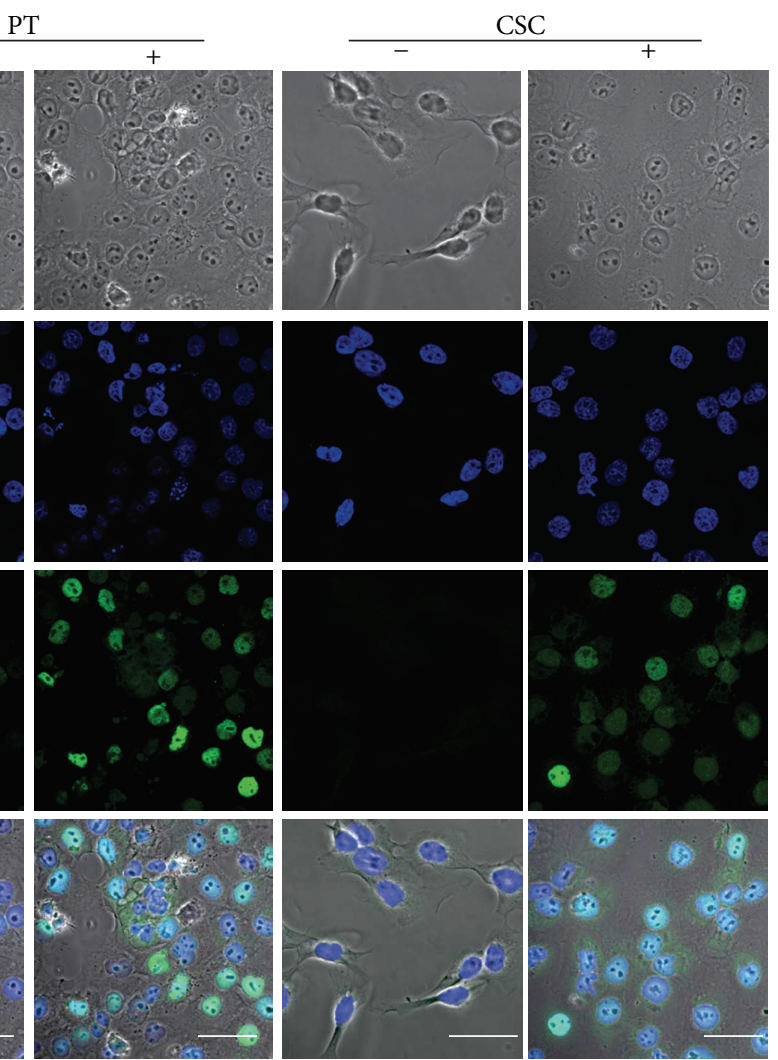

(b)

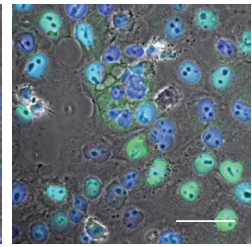

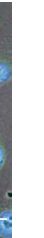

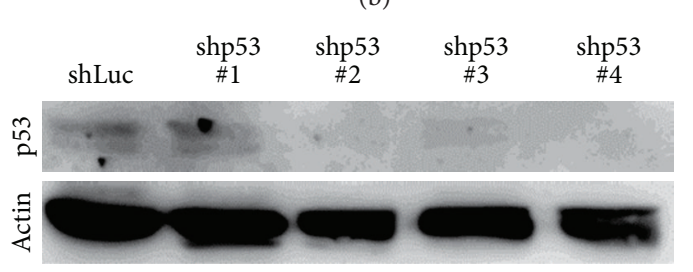

(d)

FIGURE 5: Resveratrol impeded EMT through p53 activation in CSCs. (a) Expressions of p53 and phosphorylated p53 (Ser15) were lower in TW01 CSC than those in TW01 parental cells. Resveratrol could increase expression of p53 and phosphorylated p53 expression both in CSC and parental cells. (b) Resveratrol $(50 \mu \mathrm{M})$ reactivated p53, indicated by the translocation of phosphorylated p53 (Ser15) from cytoplasm to the nucleus (detected with DAPI staining), and repressed the EMT phenotype in TW01 CSCs. TW01 parental cells remained epithelial type after resveratrol treatment. Scale bars indicate $40 \mu \mathrm{m}$. (c) Western blot indicated the p53 expression of TW01 CSCs with $50 \mu \mathrm{M}$ resveratrol treatment $(\mathrm{RV})$ or $\mathrm{p} 53$ overexpression $\left(\mathrm{p} 53^{\mathrm{ov}}\right)$. (d) Western blot confirmed the p53 knockdown efficiency in TW01 CSCs. shp53\#2 with the best knockdown efficiency was chose for further experiment.

to normal stem cells, breast CSCs contain a lower level of ROS compared with nontumorigenic cancer cells [26]. To date, the metabolic signatures of CSCs remain largely unknown. Our findings revealed that CSCs, like normal stem cells, underwent metabolic shift from aerobic metabolism to anaerobic glycolysis (Figures 2(e) and 2(f)). Moreover, the levels of ROS were lower in NPC CSCs that may result in radioresistance as previously reported in breast CSCs (Figures $1(\mathrm{~b})$ and $2(\mathrm{~g})$ ).

Abundant evidence has been accumulated to substantiate the anticancer activities of resveratrol in various human cancers [27-30]. We found that resveratrol could turn off the metabolic switch, increased the ROS level, and depolarized mitochondrial membranes in NPC CSCs (Figure 4). These alterations in metabolism occurred concomitantly with the suppression of the CSC properties including the resistance to radiotherapy and chemotherapy, self-renewal capacity, tumor initiation capacity, and metastatic potential in NPCCSCs (Figure 3). Particularly worth mentioning is that resveratrol tackled the nexus of NPC CSCs which resulted in extensive suppression of stemness, EMT, and metabolism-related genes (Figure 3(h)). This extensive suppression in CSCs could also be observed after we had ectopically expressed p53, the downstream target of resveratrol (Figure 6). In addition, the suppression of CSC properties by resveratrol could be attenuated by knocking down p53 (Figure 6). These findings 


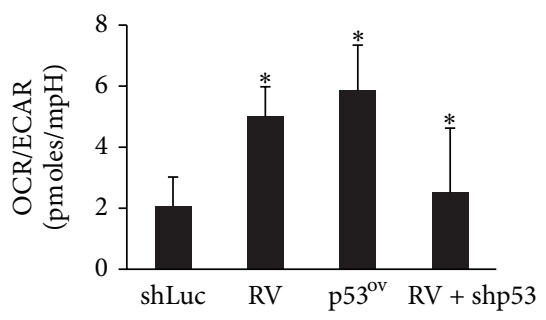

(a)

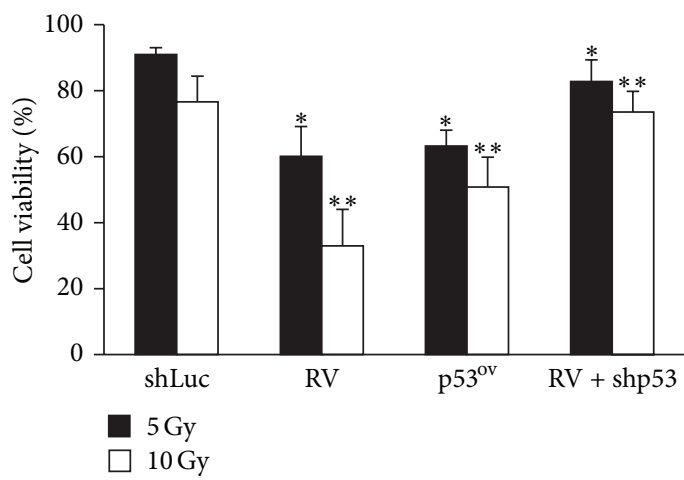

(d)

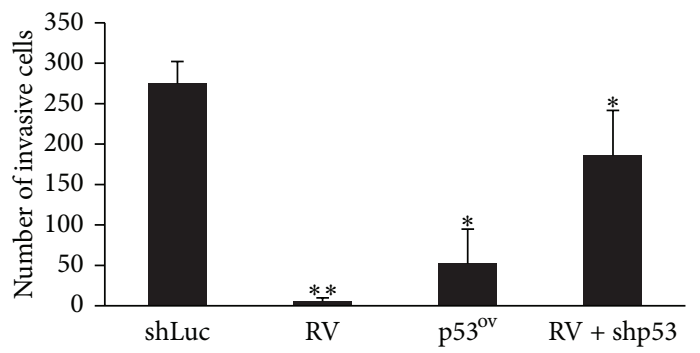

(f)
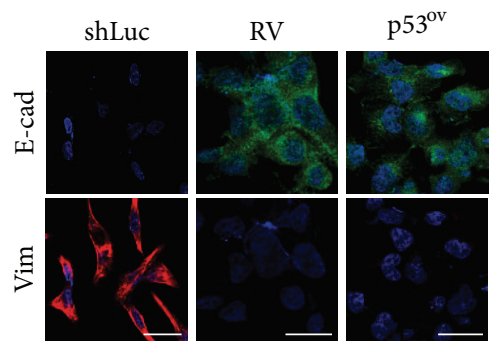

(h)

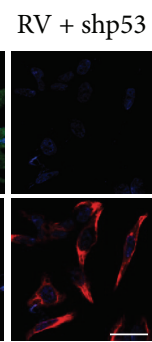

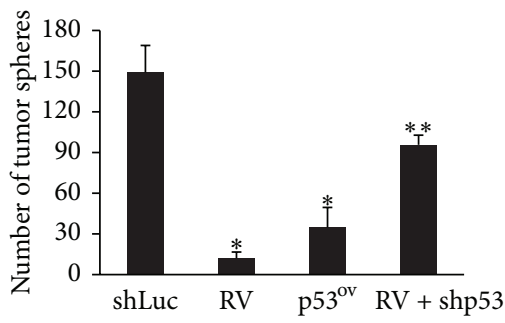

(b)

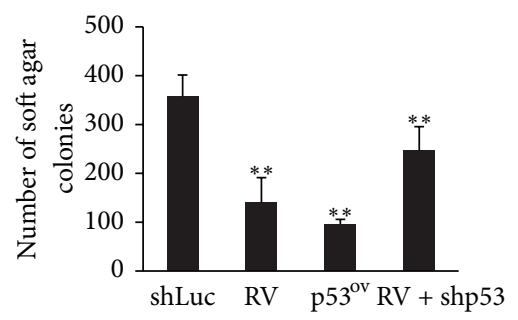

(c)

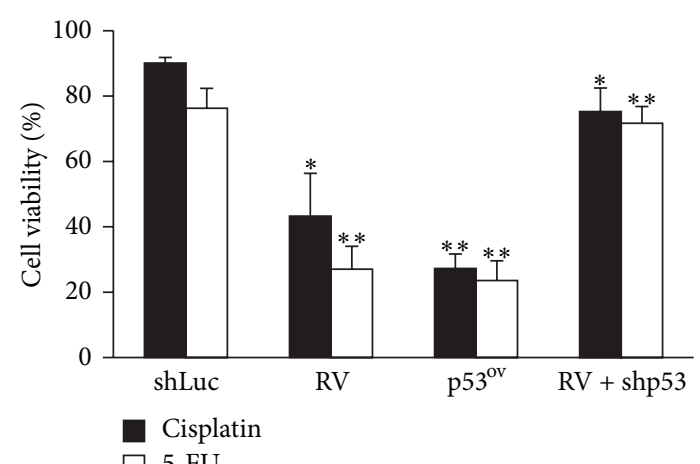

(e)

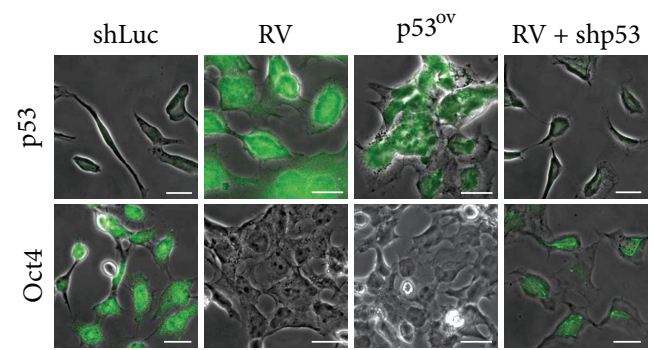

(g)

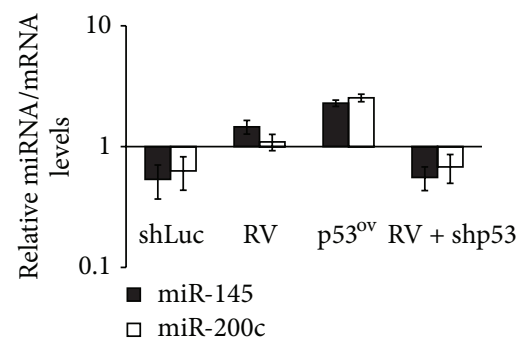

(i)

FIGURE 6: Loss of p53 expression reversed the effects of resveratrol on CSCs. Resveratrol and p53 overexpression had similar effects on TW01 CSCs in (a) OCR/ECAR ratio measurement, (b) tumor sphere formation assay, (c) soft agar assay, (d) cell viability assay under 5 Gy and 10 Gy irradiation, (e) cell viability assay under cisplatin $(8 \mu \mathrm{M})$ and 5-fluorouracil (5-FU; $10 \mu \mathrm{M})$ treatment, and (f) invasive capacity assay. Repressing p53 expression diminished resveratrol effects. (g) Resveratrol and p53 overexpression suppressed Oct4 expression in TW01 CSCs. Loss of p53 expression reversed the effects of resveratrol. (h) Similarly, resveratrol and p53 overexpression reversed EMT process with increased E-cadherin (E-cad) and decreased vimentin (Vim) expression. Loss of p53 expression reversed the effects of resveratrol. Scale bars in $(\mathrm{g})$ and $(\mathrm{h})$ indicate $25 \mu \mathrm{m}$. (i) Upregulation of miR-145 and miR-200c was detected in resveratrol-treated and p53 overexpressed CSCs. Blocking p53 expression could reverse their expressions. Results were normalized with RNU6B and compared with that in TW01 parental cells. (shLuc: CSCs with shLuc as a control; RV: CSCs with resveratrol treatment; $553^{\text {ov }}$ : CSCs with p53 overexpression; RV + shp53: resveratrol-treated CSCs with p53 knockdown; RV group and $\mathrm{p} 53^{\text {ov }}$ group were compared with shLuc group; RV + shp53 group was compared with RV group; $\left.{ }^{*} P<0.05 ;{ }^{* *} P<0.01\right)$. 


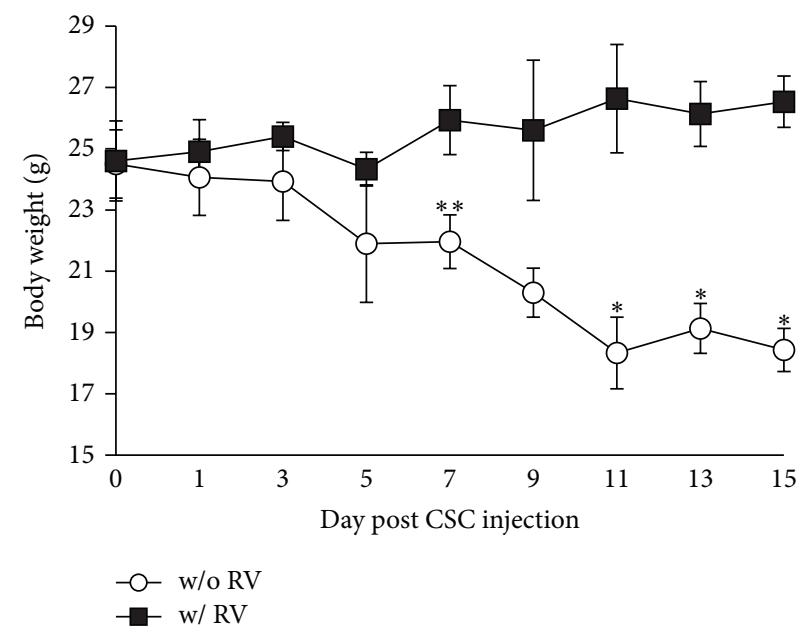

(a)
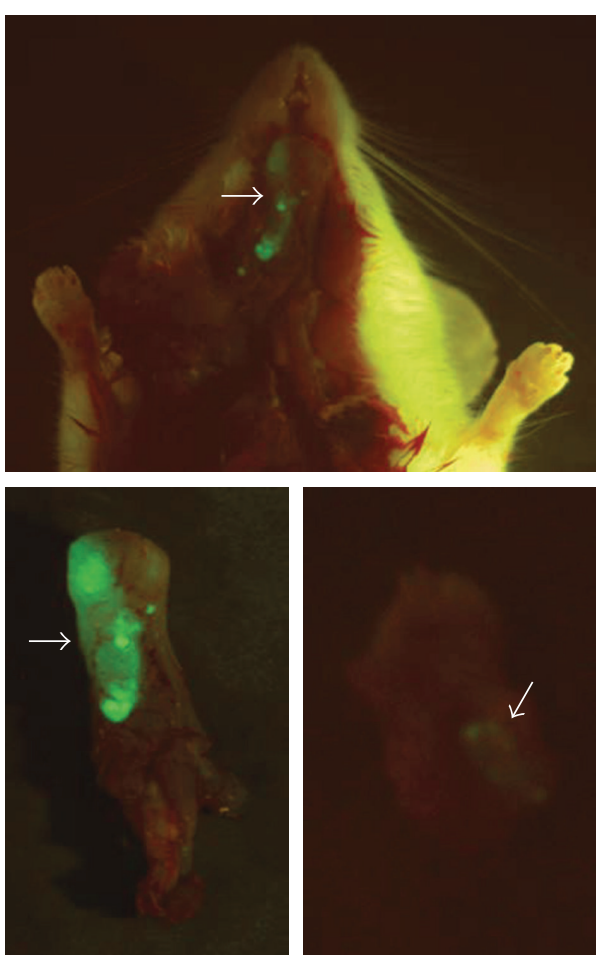

(c)

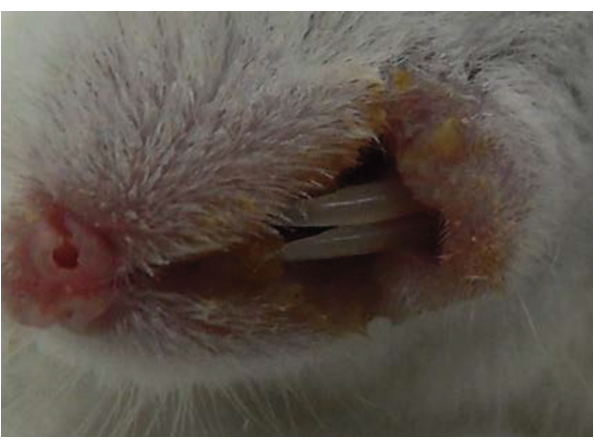

(b)

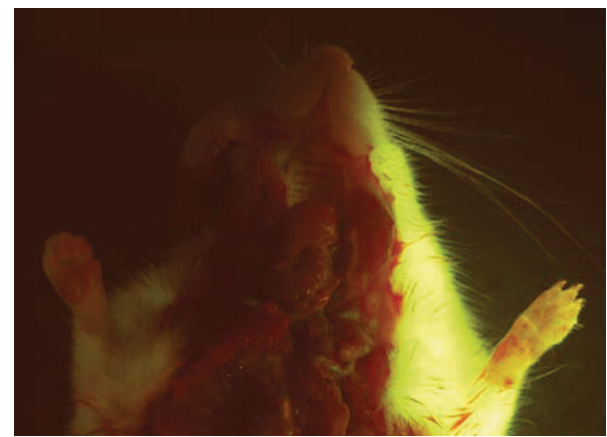

(d)

FIGURE 7: Resveratrol suppressed the in vivo growth of CSCs. (a) Mice without resveratrol feeding (w/o RV) were $6 \mathrm{~g}$ thinner in body weight as compared with the mice with resveratrol feeding (w/ RV) after 2 weeks. (b) By hypoglossal injection and without resveratrol feeding, the indurate tumor lumps disseminated around NOD/SCID mouse's buccal mucosa after two weeks. (c) By hypoglossal injection and without resveratrol feeding for two weeks, GFP-lentivirus-infected TW01 CSCs grew throughout the tongue (bottom left) and lower jaw (bottom right) of the mouse, as seen under a Sky-blue II epifluorescent light (tumor size 50-270 $\mathrm{mm}^{3}$ ). (d) As seen under a Sky-blue II epifluorescent light, TW01 CSCs were eradicated in the hypoglossal region of the mouse tube-fed with resveratrol after two weeks. $\left({ }^{* *} P<0.05\right.$; $\left.{ }^{* *} P<0.01\right)$.

substantiated the notion that $\mathrm{p} 53$ may serve as a common link between metabolism, stemness, and EMT in CSCs.

It was reported that resveratrol can increase the p53 protein level in breast cancer cell line without altering the p53 mRNA levels, suggesting that resveratrol may still be useful to treat tumors with a loss of normal p53 function [31]. Besides, resveratrol could significantly activate intracellular
Notch-1 and restore wild-type p53 expression in glioblastoma cells [32]. These findings indicate that resveratrol may be an effective drug for treatment of tumors without normal p53 function.

Recent studies have revealed a connection between p53 and stem cell biology, which signified the importance of p53 pathway in CSCs $[33,34]$. Several studies indicated that 
p53 pathway decreases the efficiency of reprogramming of somatic cells to iPSCs [35-39]. The absence of p53 allows the suboptimal cells to become iPSCs and also accelerates nuclear reprogramming by loss of p53-dependent cell cycle arrest. The iPSCs with functional p53 were able to form teratoma which differentiated into three germ layers. However, the absence of p 53 seemed to be the tradeoff between reprogramming efficiency and tumorigenesis. The teratoma of iPSCs with p53 knockout or a mutation in p53 contained undifferentiated tissue cells which resembled tumor growth [35]. Besides, p53 could control the MDM2-mediated slug degradation [40] and suppress c-Myc through the induction of miR-145 [41]. p53 also could inhibit the expression of CD44 by modulating miR-34a in prostate CSCs [42]. Moreover, p53 was able to launch miR-200c to suppress genes that mediate EMT and stemness properties [43].

These findings suggest that p53 has a significant role in connecting reprogramming to stemness status and tumorigenesis of CSCs. In ESC studies, researchers found that miR145 suppressed the expression of Oct4, Sox2, and Klf4 [44]. In cancer studies, researchers found that miR-145 could be considered as a tumor suppressor and was downregulated in several cancers including NPC [45]. Since resveratrolinduced p53 is able to transcriptionally activate the expression of miR-145 and miR-200c (Figure 6(i)), the role of p53 in suppressing the stemness and EMT via induction of miRNAs awaits further investigation. Through the elucidation of the p53/miRNAs mechanism, we may be able to apply this regulation in the therapy targeting at CSCs.

\section{Conclusions}

Thus far, the link between metabolic reprogramming, stemness, and EMT of CSCs has remained elusive. However, this link appears to be of vital importance as uncovered by the aforementioned effects of resveratrol. The regulation of p53 within stemness, EMT, and metabolic reprogramming should be further investigated with the hope that it may lead to the identification of novel therapeutic targets for future anticancer therapies in humans.

\section{Conflict of Interests}

The authors proclaim that they have no conflict of interests in conducting this study and publishing the results presented in this paper.

\section{Authors' Contribution}

Y. Shen and C. Lin contributed equally to the work.

\section{Acknowledgments}

This study was financially supported by Grants from the National Science Council, Taiwan (NSC96-2320-B-010-035MY2, NSC99-2314-B-010-006-MY3, and NSC100-2320-B010-024-MY3), the Department of Health, Taipei City Hospital (Grant no. 10001-62-030), the Ministry of Education for a Grant of Aim for the Top University Plan to the National Yang-Ming University. The authors thank the technical support provided by the Microarray \& Gene Expression Analysis Core Facility of the National Yang-Ming University (NYMU) VGH Genome Research Center. The Gene Expression Analysis Core Facility is supported by the National Research Program for Genomic Medicine, National Science Council.

\section{References}

[1] K. W. Lo, K. F. To, and D. P. Huang, "Focus on nasopharyngeal carcinoma," Cancer Cell, vol. 5, no. 5, pp. 423-428, 2004.

[2] Y. Bensouda, W. Kaikani, N. Ahbeddou et al., "Treatment for metastatic nasopharyngeal carcinoma," European Annals of Otorhinolaryngology, Head and Neck Diseases, vol. 128, no. 2, pp. 79-85, 2011.

[3] M. T. Mueller, P. C. Hermann, and C. Heeschen, "Cancer stem cells as new therapeutic target to prevent tumour progression and metastasis," Frontiers in Bioscience, vol. 2, pp. 602-613, 2010.

[4] S. A. Mani, W. Guo, M. J. Liao et al., "The epithelial-mesenchymal transition generates cells with properties of stem cells," Cell, vol. 133, no. 4, pp. 704-715, 2008.

[5] M. Ksiazkiewicz, A. Markiewicz, and A. J. Zaczek, "Epithelialmesenchymal transition: a hallmark in metastasis formation linking circulating tumor cells and cancer stem cells," Pathobiology, vol. 79, no. 4, pp. 195-208, 2012.

[6] D. Fong, A. Yeh, R. Naftalovich, T. H. Choi, and M. M. Chan, "Curcumin inhibits the side population (SP) phenotype of the rat C6 glioma cell line: towards targeting of cancer stem cells with phytochemicals," Cancer Letters, vol. 293, no. 1, pp. 65-72, 2010.

[7] S. N. Tang, C. Singh, D. Nall, D. Meeker, S. Shankar, and R. K. Srivastava, "The dietary bioflavonoid quercetin synergizes with epigallocathechin gallate (EGCG) to inhibit prostate cancer stem cell characteristics, invasion, migration and epithelialmesenchymal transition," Journal of Molecular Signaling, vol. 5, article 14, 2012.

[8] C. H. Lin, Y. A. Shen, P. H. Hung, Y. B. Yu, and Y. J. Chen, "Epigallocathechin gallate, polyphenol present in green tea, inhibits stem-like characteristics and epithelial-mesenchymal transition in nasopharyngeal cancer cell lines," BMC Complementary and Alternative Medicine, vol. 12, no. 201, pp. 1-12, 2012.

[9] F. W. Hu, L. L. Tsai, C. H. Yu et al., "Impairment of tumorinitiating stem-like property and reversal of epithelial-mesenchymal transdifferentiation in head and neck cancer by resveratrol treatment," Molecular Nutrition \& Food Research, vol. 56, no. 8, pp. 1247-1258, 2012.

[10] T. T. Huang, H. C. Lin, C. C. Chen et al., "Resveratrol induces apoptosis of human nasopharyngeal carcinoma cells via activation of multiple apoptotic pathways," Journal of Cellular Physiology, vol. 226, no. 3, pp. 720-728, 2011.

[11] S. Shankar, D. Nall, S. N. Tang et al., "Resveratrol inhibits pancreatic cancer stem cell characteristics in human and KrasG12D transgenic mice by inhibiting pluripotency maintaining factors and epithelial-mesenchymal transition," PLOS ONE, vol. 6, no. 1, Article ID e16530, 2011.

[12] P. R. Pandey, H. Okuda, M. Watabe et al., "Resveratrol suppresses growth of cancer stem-like cells by inhibiting fatty acid synthase," Breast Cancer Research and Treatment, vol. 130, no. 2, pp. 387-398, 2011. 
[13] Y. P. Yang, Y. L. Chang, P. I. Huang et al., "Resveratrol suppresses tumorigenicity and enhances radiosensitivity in primary glioblastoma tumor initiating cells by inhibiting the STAT3 axis," Journal of Cellular Physiology, vol. 227, no. 3, pp. 976-993, 2011.

[14] H. Wang, H. Zhang, L. Tang et al., "Resveratrol inhibits TGF-betal-induced epithelial-to-mesenchymal transition and suppresses lung cancer invasion and metastasis," Toxicology, vol. 303, pp. 139-146, 2013.

[15] D. Vergara, C. M. Valente, A. Tinelli et al., "Resveratrol inhibits the epidermal growth factor-induced epithelial mesenchymal transition in MCF-7 cells," Cancer Letters, vol. 310, no. 1, pp. 1-8, 2011.

[16] C. T. Lin, W. Y. Chan, W. Chen et al., "Characterization of seven newly established nasopharyngeal carcinoma cell lines," Laboratory Investigation, vol. 68, no. 6, pp. 716-727, 1993.

[17] R. Glaser, H. Y. Zhang, K. Yao et al., "Two epithelial tumor cell lines (HNE-1 and HONE-1) latently infected with EpsteinBarr virus that were derived from nasopharyngeal carcinomas," Proceedings of the National Academy of Sciences of the United States of America, vol. 86, no. 23, pp. 9524-9528, 1989.

[18] J. T. C. Chang, S. H. Chan, C. Y. Lin et al., "Differentially expressed genes in radioresistant nasopharyngeal cancer cells: gp96 and GDF15," Molecular Cancer Therapeutics, vol. 6, no. 8, pp. 2271-2279, 2007.

[19] S. F. Chen, Y. C. Chang, S. Nieh et al., "Nonadhesive culture system as a model of rapid sphere formation with cancer stem cell properties," PLoS ONE, vol. 7, no. 2, Article ID e31864, 2012.

[20] J. Wang, L. P. Guo, L. Z. Chen, Y. X. Zeng, and H. L. Shih, "Identification of cancer stem cell-like side population cells in human nasopharyngeal carcinoma cell line," Cancer Research, vol. 67, no. 8, pp. 3716-3724, 2007.

[21] C. S. Lin, H. T. Lee, S. Y. Lee et al., "High mitochondrial DNA copy number and bioenergetic function are associated with tumor invasion of esophageal squamous cell carcinoma cell lines,' International Journal of Molecular Sciences, vol. 13, no. 9, pp. 11228-11246, 2012.

[22] S. Sethi, N. M. Radio, M. P. Kotlarczyk et al., "Determination of the minimal melatonin exposure required to induce osteoblast differentiation from human mesenchymal stem cells and these effects on downstream signaling pathways," Journal of Pineal Research, vol. 49, no. 3, pp. 222-238, 2010.

[23] W. Qian and B. Van Houten, "Alterations in bioenergetics due to changes in mitochondrial DNA copy number," Methods, vol. 51, no. 4, pp. 452-457, 2010.

[24] O. Warburg, "On the origin of cancer cells," Science, vol. 123, no. 3191, pp. 309-314, 1956.

[25] A. Prigione, B. Fauler, R. Lurz, H. Lehrach, and J. Adjaye, "The senescence-related mitochondrial/oxidative stress pathway is repressed in human induced pluripotent stem cells," Stem Cells, vol. 28 , no. 4, pp. 721-733, 2010.

[26] M. Diehn, R. W. Cho, N. A. Lobo et al., "Association of reactive oxygen species levels and radioresistance in cancer stem cells," Nature, vol. 458, no. 7239, pp. 780-783, 2009.

[27] P. L. Kuo, L. C. Chiang, and C. C. Lin, "Resveratrol- induced apoptosis is mediated by p53-dependent pathway in Hep G2 cells," Life Sciences, vol. 72, no. 1, pp. 23-34, 2002.

[28] M. J. Atten, E. Godoy-Romero, B. M. Attar, T. Milson, M. Zopel, and O. Holian, "Resveratrol regulates cellular PKC $\alpha$ and $\delta$ to inhibit growth and induce apoptosis in gastric cancer cells," Investigational New Drugs, vol. 23, no. 2, pp. 111-119, 2005.
[29] C. L. Kao, P. I. Huang, P. H. Tsai et al., "Resveratrol-induced apoptosis and increased radiosensitivity in CD133-positive cells derived from atypical teratoid/rhabdoid tumor," International Journal of Radiation Oncology Biology Physics, vol. 74, no. 1, pp. 219-228, 2009.

[30] L. J. Yu, M. L. Wu, H. Li et al., "Inhibition of STAT3 expression and signaling in resveratrol-differentiated medulloblastoma cells," Neoplasia, vol. 10, no. 7, pp. 736-744, 2008.

[31] D. C. F. da Costa, F. A. Casanova, J. Quarti et al., "Transient transfection of a wild-type p53 gene triggers resveratrolinduced apoptosis in cancer cells," PLOS ONE, vol. 7, no. 11, Article ID e48746, 2012.

[32] H. Lin, W. Xiong, X. Zhang et al., "Notch-1 activation-dependent p53 restoration contributes to resveratrol-induced apoptosis in glioblastoma cells," Oncology Reports, vol. 26, no. 4, pp. 925-930, 2011.

[33] V. V. Prabhu, J. E. Allen, B. Hong et al., “Therapeutic targeting of the p53 pathway in cancer stem cells," Expert Opinion Therapeutic Targets, vol. 16, no. 12, pp. 1161-1174, 2012.

[34] C. Ginestier, E. Charafe-Jauffret, and D. Birnbaum, "p53 and cancer stem cells: the mevalonate connexion," Cell Cycle, vol. 11, no. 14, pp. 2583-2584, 2012.

[35] H. Hong, K. Takahashi, T. Ichisaka et al., "Suppression of induced pluripotent stem cell generation by the p53-p21 pathway," Nature, vol. 460, no. 7259, pp. 1132-1135, 2009.

[36] H. Li, M. Collado, A. Villasante et al., "The Ink4/Arf locus is a barrier for iPS cell reprogramming," Nature, vol. 460, no. 7259, pp. 1136-1139, 2009.

[37] T. Kawamura, J. Suzuki, Y. V. Wang et al., "Linking the p53 tumour suppressor pathway to somatic cell reprogramming," Nature, vol. 460, no. 7259, pp. 1140-1144, 2009.

[38] J. Utikal, J. M. Polo, M. Stadtfeld et al., "Immortalization eliminates a roadblock during cellular reprogramming into iPS cells," Nature, vol. 460, no. 7259, pp. 1145-1148, 2009.

[39] R. M. Marión, K. Strati, H. Li et al., "A p53-mediated DNA damage response limits reprogramming to ensure iPS cell genomic integrity," Nature, vol. 460, no. 7259, pp. 1149-1153, 2009.

[40] S. P. Wang, W. L. Wang, Y. L. Chang et al., "p53 controls cancer cell invasion by inducing the MDM2-mediated degradation of Slug," Nature Cell Biology, vol. 11, no. 6, pp. 694-704, 2009.

[41] M. Sachdeva, S. Zhu, F. Wu et al., "p53 represses c-Myc through induction of the tumor suppressor miR-145," Proceedings of the National Academy of Sciences of the United States of America, vol. 106, no. 9, pp. 3207-3212, 2009.

[42] C. Liu, K. Kelnar, B. Liu et al., "The microRNA miR-34a inhibits prostate cancer stem cells and metastasis by directly repressing CD44," Nature Medicine, vol. 17, no. 2, pp. 211-215, 2011.

[43] C. J. Chang, C. H. Chao, W. Xia et al., "P53 regulates epithelialmesenchymal transition and stem cell properties through modulating miRNAs," Nature Cell Biology, vol. 13, no. 3, pp. 317-323, 2011.

[44] N. Xu, T. Papagiannakopoulos, G. Pan, J. A. Thomson, and K. S. Kosik, "MicroRNA-145 regulates OCT4, SOX2, and KLF4 and represses pluripotency in human embryonic stem cells," Cell, vol. 137, no. 4, pp. 647-658, 2009.

[45] H. C. Chen, G. H. Chen, Y. H. Chen et al., "MicroRNA deregulation and pathway alterations in nasopharyngeal carcinoma," British Journal of Cancer, vol. 100, no. 6, pp. 1002-1011, 2009. 


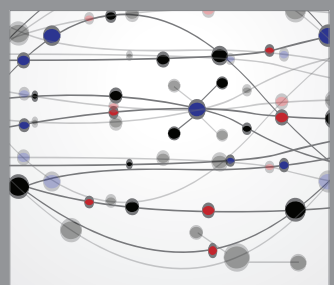

The Scientific World Journal
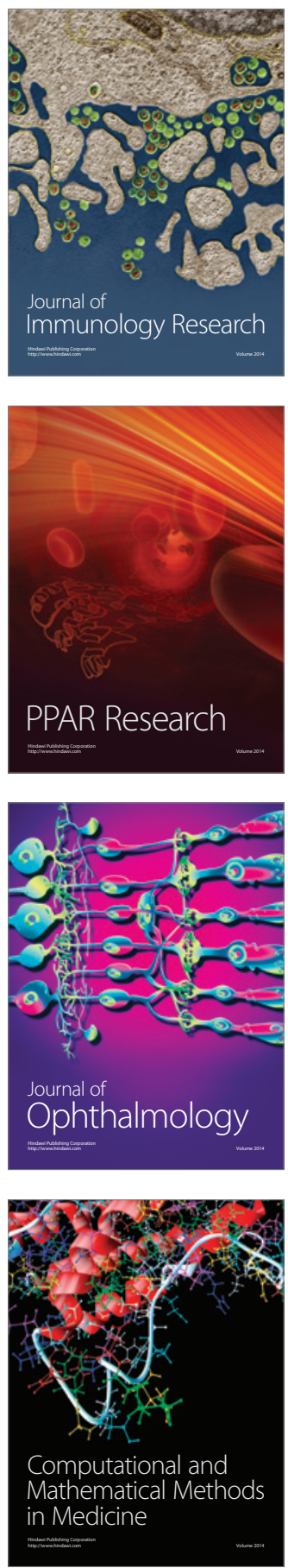

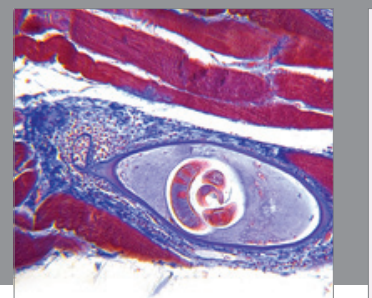

Gastroenterology

Research and Practice
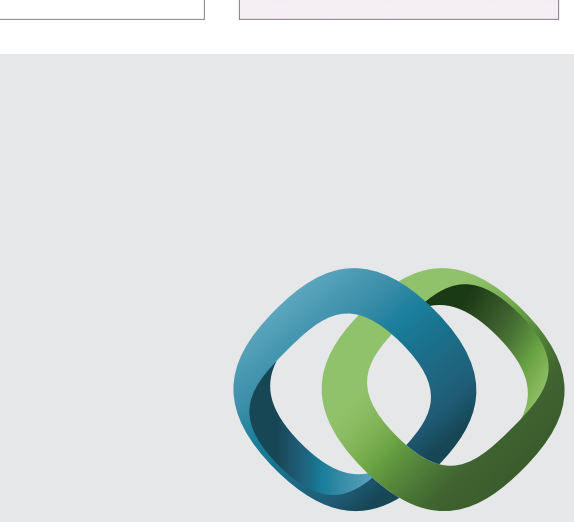

\section{Hindawi}

Submit your manuscripts at

http://www.hindawi.com
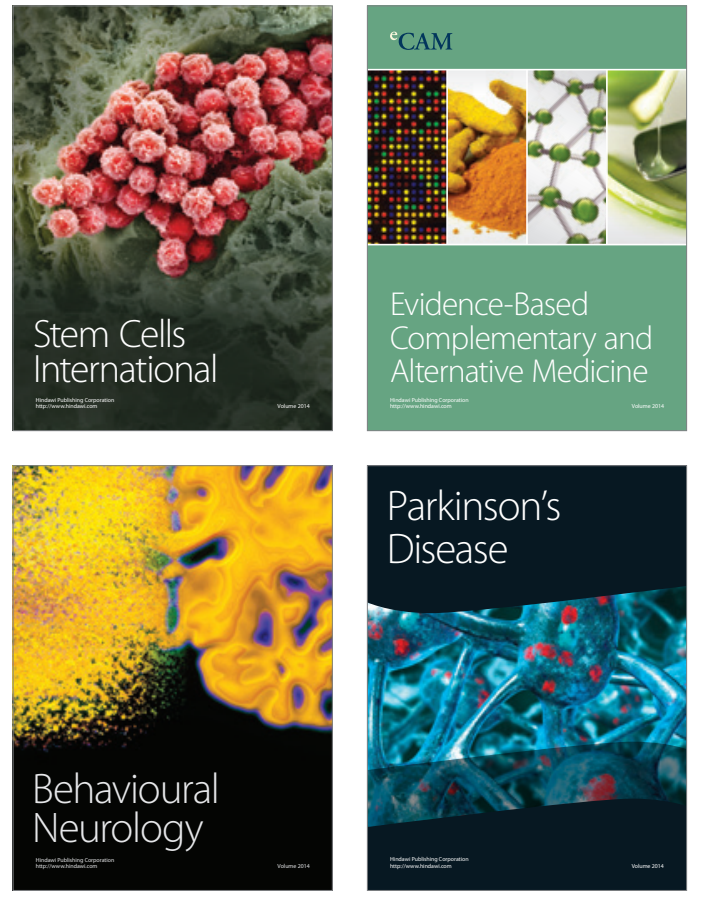
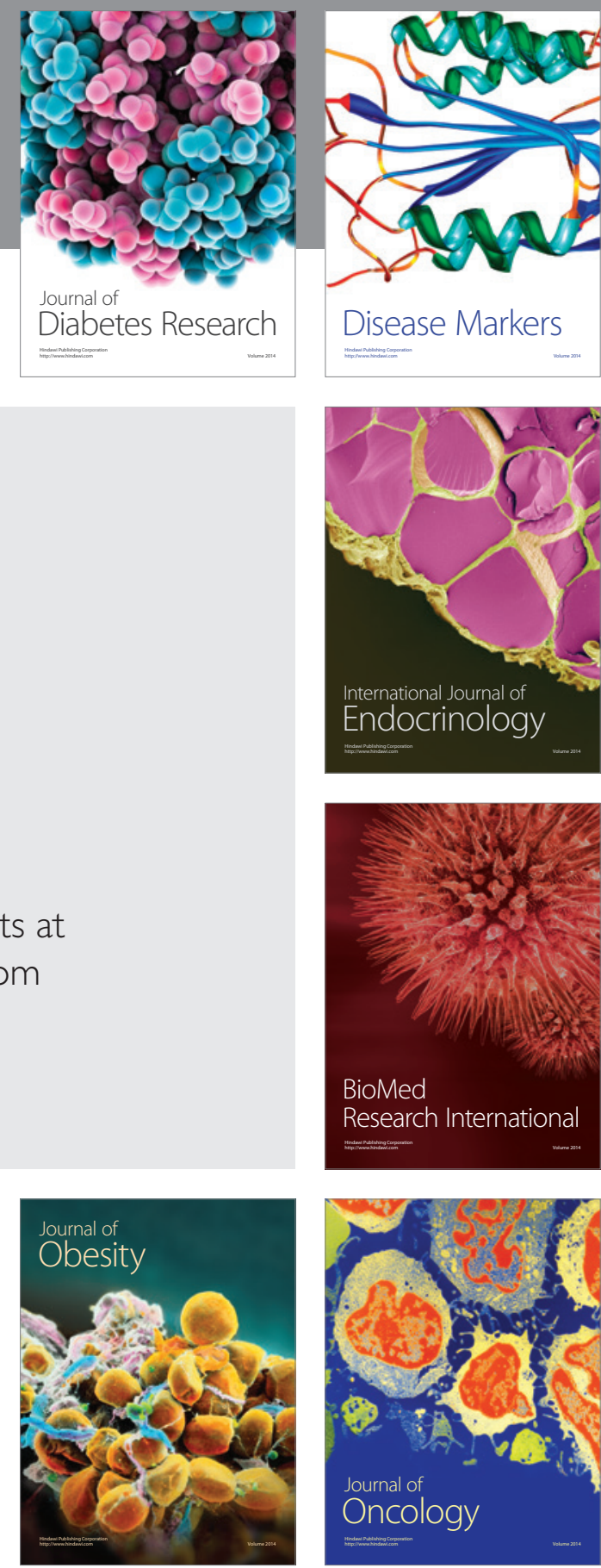

Disease Markers
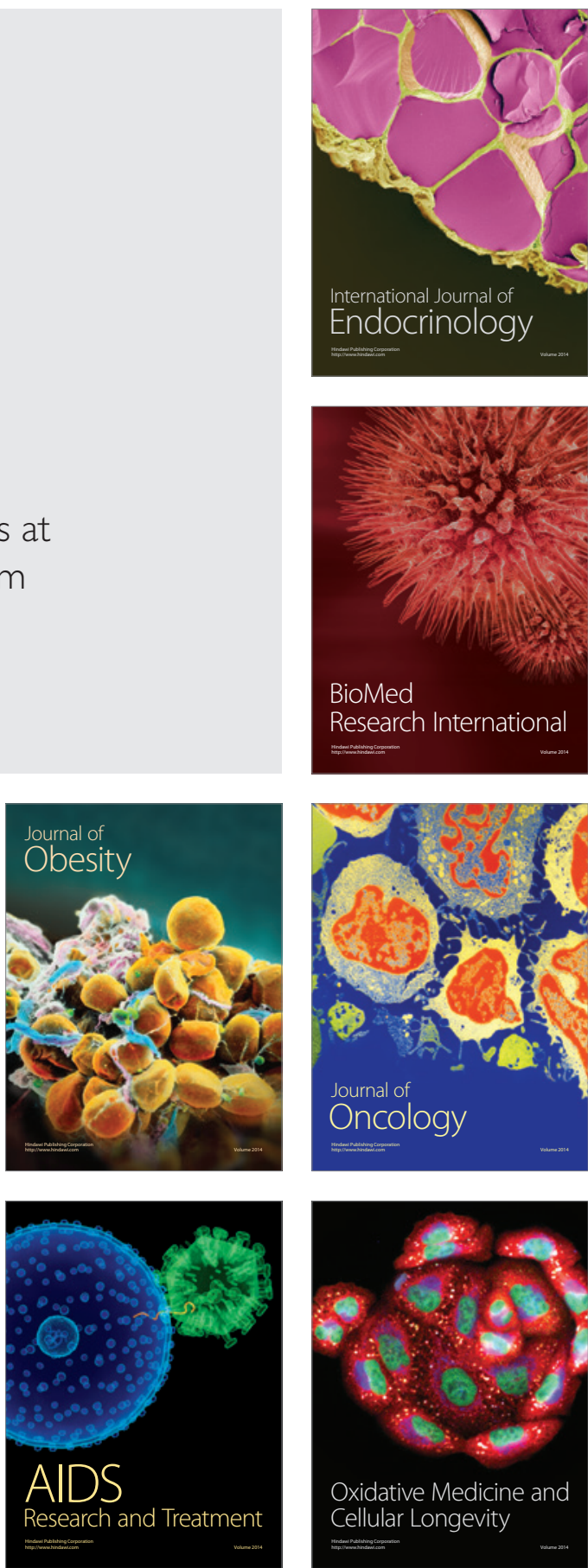